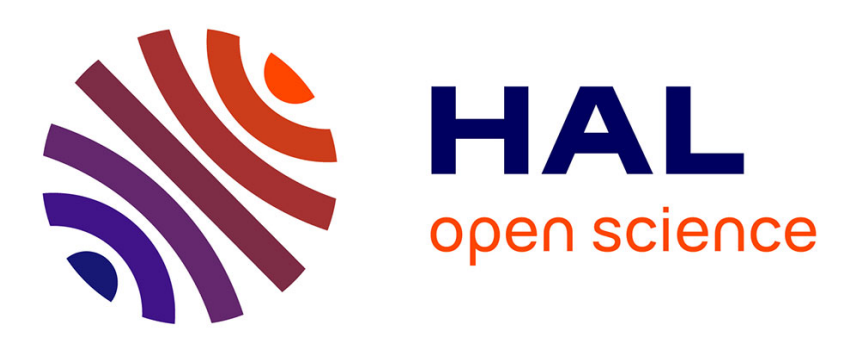

\title{
On some graph classes related to perfect graphs: A survey
}

\author{
Flavia Bonomo-Braberman, Guillermo Durán, Martín D Safe, Annegret K
} Wagler

\section{- To cite this version:}

Flavia Bonomo-Braberman, Guillermo Durán, Martín D Safe, Annegret K Wagler. On some graph classes related to perfect graphs: A survey. Discrete Applied Mathematics, 2020, 281, pp.42-60. 10.1016/j.dam.2019.05.019 . hal-03142102

\author{
HAL Id: hal-03142102 \\ https://hal.uca.fr/hal-03142102
}

Submitted on 15 Feb 2021

HAL is a multi-disciplinary open access archive for the deposit and dissemination of scientific research documents, whether they are published or not. The documents may come from teaching and research institutions in France or abroad, or from public or private research centers.
L'archive ouverte pluridisciplinaire HAL, est destinée au dépôt et à la diffusion de documents scientifiques de niveau recherche, publiés ou non, émanant des établissements d'enseignement et de recherche français ou étrangers, des laboratoires publics ou privés. 


\title{
On graph classes related to perfect graphs:
} A survey

\author{
Flavia Bonomo ${ }^{1}$, Guillermo Durán ${ }^{2}$, Martín D. Safe ${ }^{3}$, and \\ Annegret K. Wagler ${ }^{4}$ \\ ${ }^{1}$ Universidad de Buenos Aires. Facultad de Ciencias Exactas y \\ Naturales. Departamento de Computación. / \\ CONICET-Universidad de Buenos Aires. Instituto de \\ Investigación en Ciencias de la Computación (ICC). Buenos \\ Aires, Argentina,fbonomo@dc.uba.ar \\ ${ }^{2}$ Universidad de Buenos Aires. Facultad de Ciencias Exactas y \\ Naturales. Departamento de Matemática. / \\ CONICET-Universidad de Buenos Aires. Instituto de Cálculo \\ (IC). Buenos Aires, Argentina / Departamento de Ingeniería \\ Industrial, Facultad de Ciencias Físicas y Matemáticas, \\ Universidad de Chile, Santiago, Chile,gduran@dm.uba.ar \\ ${ }^{3}$ Departamento de Matemática, Universidad Nacional del Sur, \\ Bahía Blanca, Argentina / INMABB (UNS-CONICET), Bahía \\ Blanca, Argentina,msafe@uns.edu.ar \\ ${ }^{4}$ Laboratoire d'Informatique, de Modélisation et d'Optimisation des \\ Systèmes (LIMOS, UMR CNRS 6158), Université Clermont \\ Auvergne, Clermont-Ferrand, France, annegret.wagler@uca.fr
}

\begin{abstract}
Perfect graphs form a well-known class of graphs introduced by Berge in the 1960s in terms of a min-max type equality involving two famous graph parameters. In this work, we study variants and subclasses of perfect graphs defined by means of min-max relations of other graph parameters. Our focus is on clique-perfect, coordinated, and neighborhoodperfect graphs. We show the connection between graph classes and both hypergraph theory and the clique graph operator. We review different partial characterizations of them by forbidden induced subgraphs, present the previous results, and the main open problems. Computational complexity problems are also discussed.
\end{abstract}




\section{Introduction}

Perfect graphs were defined by Berge in the 1960s in terms of a min-max type equality involving two important parameters: the clique-number and the chromatic number. Coloring a graph is the task of assigning colors to its vertices in such a way that no two adjacent vertices receive the same color. In many situations we are interested in knowing the minimum number of different colors needed to color a certain graph $G$. This minimum number is called the chromatic number of $G$ and is denoted by $\chi(G)$. A complete is a set of vertices that are pairwise adjacent and a clique is a complete set that is not properly contained in any other. The maximum cardinality of a clique of a graph $G$ is called the clique number of $G$ and is denoted by $\omega(G)$. Clearly, in any coloring, the vertices of a clique must receive different colors. Thus, $\omega(G)$ is a trivial lower bound for $\chi(G)$, i.e., the min-max type inequality

$$
\omega(G) \leq \chi(G) \text { holds for any graph } G .
$$

Moreover, the difference between $\chi(G)$ and $\omega(G)$ can be arbitrarily large. Mycielski presented in [61] a family of graphs $\left\{G_{n}\right\}_{n \geq 2}$ with $\omega\left(G_{n}\right)=2$ and $\chi\left(G_{n}\right)=n$. In this context, Berge defined a graph $G$ to be perfect if and only if the min-max type equality $\omega\left(G^{\prime}\right)=\chi\left(G^{\prime}\right)$ holds for each induced subgraph $G^{\prime}$ of $G$.

Min-max type relations play a remarkable role in the field of discrete mathematics. In the following pages, we will recall two famous min-max type theorems due to König for bipartite graphs. Other notable examples are Dilworth's theorem [31] that dictates that in any partial order the maximum size of an antichain equals the minimum number of chains needed to cover it, Menger's theorem [60] that states that the maximum number of disjoint paths joining two vertices $s$ and $t$ equals the minimum number of edges in an st-cut, and its generalization, the max-flow min-cut theorem [40] that ensures that the maximum amount of flow in a network equals the capacity of a minimum cut.

The complement of a graph $G$ is the graph $\bar{G}$ whose vertex set is the same as the vertex set of $G$ but such that any pair of different vertices are adjacent in $\bar{G}$ if and only if they are nonadjacent in $G$. In 1972, Lovász, and shortly after Fulkerson, proved a conjecture by Berge stating the following:

Theorem 1 (Perfect Graph Theorem [56]) A graph is perfect if and only if its complement is perfect.

A stable set of a graph is a set of vertices that are pairwise nonadjacent. The stability number of a graph $G$ is the maximum cardinality $\alpha(G)$ of a stable set of $G$. The clique covering number of a graph $G$ is defined as the minimum number of cliques of $G$ needed to cover the vertices of $G$, and it is denoted by $\theta(G)$. Clearly, $\alpha(G) \leq \theta(G)$. Moreover, $\alpha(G)=\omega(\bar{G})$ and $\theta(G)=\chi(\bar{G})$. Therefore, by the Perfect Graph Theorem, the notion of perfection can also be formulated in terms of a min-max type equality involving the stability number and the 
clique covering number: a graph $G$ is perfect if and only if $\alpha\left(G^{\prime}\right)=\theta\left(G^{\prime}\right)$ for each induced subgraph $G^{\prime}$ of $G$.

A hole is a chordless cycle of length at least 5 (a chord is an edge joining two nonconsecutive vertices of the cycle). An antihole is the complement of a hole. A hole, or antihole, is said odd or even if it has an odd or an even number of vertices. We say that a graph has an odd hole (resp. antihole) if it contains an induced odd hole (resp. antihole). A hole of length $n$ is denoted by $C_{n}$.

It is not difficult to verify that odd holes and odd antiholes are imperfect (i.e., not perfect). Since the class of perfect graphs is hereditary, any perfect graph has no odd holes and no odd antiholes. Furthermore, Berge conjectured, and Chudnovsky, Robertson, Seymour, and Thomas proved the following forbidden induced subgraph characterization for perfect graphs:

Theorem 2 (Strong Perfect Graph Theorem (SPGT) [24]) A graph G is perfect if and only if $G$ has no odd hole and no odd antihole.

Shortly before, Chudnovsky, Cornuéjols, Liu, Seymour, and Vušković devised a polynomial-time algorithm for recognizing perfect graphs [23].

The class of clique-perfect graphs is defined in a somewhat similar fashion. A clique-independent set of a graph $G$ is a subset of pairwise disjoint cliques of $G$. A clique-transversal of $G$ is a subset of vertices intersecting all the cliques of $G$. Denote by $\alpha_{\mathrm{c}}(G)$ and $\tau_{\mathrm{c}}(G)$ the maximum cardinality of a clique-independent set and the minimum cardinality of a clique-transversal of $G$, respectively. Clearly, the min-max type inequality

$$
\alpha_{\mathrm{c}}(G) \leq \tau_{\mathrm{c}}(G) \text { holds for any graph } G \text {. }
$$

In analogy to perfect graphs, a graph $G$ is said to be clique-perfect if and only if $\alpha_{\mathrm{c}}\left(G^{\prime}\right)=\tau_{\mathrm{c}}\left(G^{\prime}\right)$ holds for each induced subgraph $G^{\prime}$ of $G$. A graph that is not clique-perfect is said clique-imperfect. It is important to mention that clique-perfect graphs do not need to be perfect since, for instance, odd antiholes of length $6 n+3$ are clique-perfect for each $n \geq 1$ (Reed, 2001, cf. [36]). The difference between $\alpha_{\mathrm{c}}(G)$ and $\tau_{\mathrm{c}}(G)$ can be arbitrarily large. Durán, Lin, and Szwarcfiter presented in [36] a family of graphs $\left\{G_{n}\right\}_{n \geq 2}$ such that $\alpha_{\mathrm{c}}\left(G_{n}\right)=1$ and $\tau_{\mathrm{c}}\left(G_{n}\right)=n$ where the number of vertices of $G_{n}$ grows exponentially. Later, Lakshmanan S. and Vijayakumar [51] found another family of graphs $\left\{H_{n}\right\}_{n>1}$ such that $\alpha_{\mathrm{c}}\left(H_{n}\right)=2 n+1$ and $\tau_{\mathrm{c}}\left(H_{n}\right)=3 n+1$ but $H_{n}$ has only $5 n+2$ vertices.

The equality between $\alpha_{\mathrm{c}}(G)$ and $\tau_{\mathrm{c}}(G)$ has been implicitly studied in the literature for long time, but the name 'clique-perfect' was first introduced by Guruswami and Pandu Rangan [47]. Some well-known graph classes being cliqueperfect are: balanced graphs [7], comparability graphs [2], dually chordal graphs [19], complements of acyclic graphs [9], and distance-hereditary graphs [52].

A matching of a graph $G$ is a set of edges that pairwise do not share endpoints. A vertex cover of $G$ is a set $S$ of vertices of $G$ such that every edge of $G$ has at least one endpoint in $S$. The matching number $\nu(G)$ is the maximum cardinality of a matching of $G$, and the vertex covering number $\tau(G)$ is the 
minimum cardinality of a vertex cover. König's matching theorem [49] asserts that the min-max equality

$$
\nu(G)=\tau(G) \text { holds for any bipartite graph } G \text {. }
$$

Notice that if $G$ is bipartite and with no isolated vertices then the cliques of $G$ coincide with its edges and, as a consequence, $\alpha_{\mathrm{c}}(G)=\nu(G)$ and $\tau_{\mathrm{c}}(G)=\tau(G)$. Then, by König's matching theorem, if $G$ is bipartite and without isolated vertices then $\alpha_{\mathrm{c}}(G)=\tau_{\mathrm{c}}(G)$. It is easily seen that this equality holds even if the bipartite graph $G$ is permitted to have isolated vertices. Since the induced subgraphs of a bipartite graph are also bipartite then bipartite graphs are cliqueperfect. Thus, bipartite graphs can be regarded as a special type of cliqueperfect graphs.

Coordinated graphs form a subclass of perfect graphs and are defined similarly. Let $G$ be a graph, let $\gamma_{\mathrm{c}}(G)$ be the minimum number of colors needed to color the cliques of $G$ in such a way that two intersecting cliques receive different colors, and let $\Delta_{\mathrm{c}}(G)$ be the maximum cardinality of a family of cliques all of which have at least one vertex of $G$ in common. Clearly,

$$
\Delta_{\mathrm{c}}(G) \leq \gamma_{\mathrm{c}}(G) \text { holds for any graph } G
$$

Parameters $\Delta_{\mathrm{c}}$ and $\gamma_{\mathrm{c}}$ are generally denoted in the literature by $M$ and $F$, respectively.

A graph $G$ is called coordinated if $\Delta_{c}\left(G^{\prime}\right)=\gamma_{c}\left(G^{\prime}\right)$ for each induced subgraph $G^{\prime}$ of $G$. Since the class of coordinated graphs is hereditary by definition, and since odd holes and odd antiholes are not coordinated [12] then, by the SPGT, coordinated graphs are perfect. Also in this case, the difference between the parameters can be arbitrarily large. In fact, in [12] it is shown that, for antiholes, the difference $\gamma_{\mathrm{c}}\left(\overline{C_{n}}\right)-\Delta_{\mathrm{c}}\left(\overline{C_{n}}\right)$ grows exponentially in $n$.

If $G$ is a triangle-free graph without isolated vertices, the cliques of $G$ coincide with the edges, then $\gamma_{\mathrm{c}}(G)$ coincides with $\gamma(G)$, the so called chromatic index (minimum number of colors to color the edges of a graph so that edges that share an endpoint receive different colors), and the parameter $\Delta_{\mathrm{c}}(G)$ coincides with the maximum degree $\Delta(G)$ of the vertices of $G$. By König's edge coloring theorem [49],

$$
\gamma(G)=\Delta(G) \text { holds for any bipartite graph } G \text {. }
$$

As in the case of clique-perfection, we can conclude that $\gamma_{\mathrm{c}}(G)=\Delta_{\mathrm{c}}(G)$ holds for any bipartite graph $G$ and hence bipartite graphs are coordinated. Thus, coordinated graphs constitute another way of generalizing bipartite graphs.

Neighborhood-perfect graphs were defined in [53], also by the equality of two parameters for all induced subgraphs. Given a graph $G$, a set $C \subseteq V(G)$ is a neighborhood-covering set (or neighborhood set) if each edge and each vertex of $G$ belongs to $G[v]$ for some $v \in C$, where $G[v]$ denotes the subgraph of $G$ induced by the closed neighborhood of the vertex $v$. Two elements of $E(G) \cup V(G)$ are neighborhood-independent if there is no vertex $v \in V(G)$ such that both 


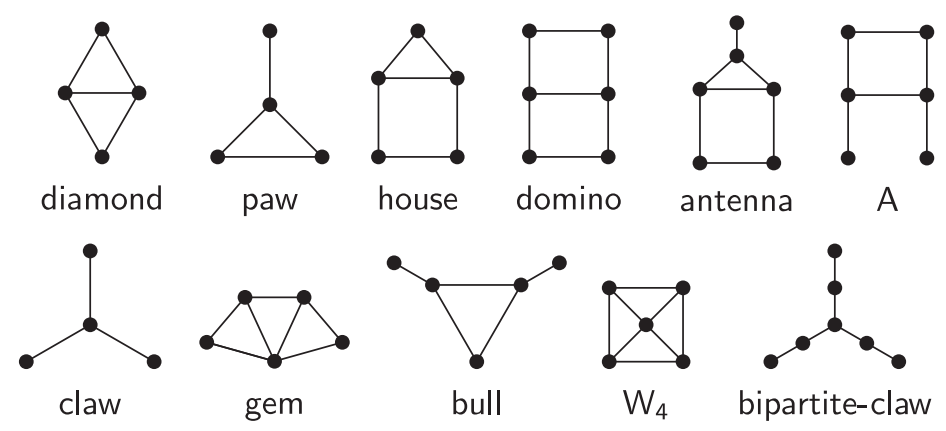

Figure 1: Some small graphs

elements are in $G[v]$. A set $S \subseteq V(G) \cup E(G)$ is said to be a neighborhoodindependent set if every pair of elements of $S$ is neighborhood-independent. Let $\rho_{\mathrm{n}}$ be the size of a minimum neighborhood-covering set and $\alpha_{\mathrm{n}}(G)$ of a maximum neighborhood-independent set. Clearly, $\rho_{\mathrm{n}} \geq \alpha_{\mathrm{n}}(G)$ for every graph $G$. When $\rho_{\mathrm{n}}\left(G^{\prime}\right)=\alpha_{\mathrm{n}}\left(G^{\prime}\right)$ for every induced subgraph $G^{\prime}$ of $G, G$ is called a neighborhood-perfect graph. It was proved in [53] that odd holes and odd antiholes are not neighborhood-perfect and hence, the Strong Perfect Graph Theorem implies that neighborhood-perfect graphs are also perfect.

In Section 2 we present the basic definitions and preliminary results. In Section 3 we discuss the connection between our subject and hypergraph theory. In Section 4 we study how clique-perfection and coordination depend on properties of the clique graph. In Section 5 the studied variants of perfect graphs are analyzed when restricted to different graph classes, detailing the previous results and providing some new contributions and open problems.

\section{Definitions and preliminaries}

All graphs in this paper are undirected, without loops and without multiple edges. We denote the vertex set of the graph $G$ by $V(G)$, and the edge set by $E(G)$. For any set $S,|S|$ will denote its cardinality. $C_{n}$ will denote the chordless cycle with $n$ vertices, $P_{n}$ the chordless path with $n$ vertices, and $K_{n}$ a complete with $n$ vertices. Path and cycles are assumed to be simple (i.e., with no repeated vertices aside from the starting and ending vertices in the case of cycles). A cycle of a graph is Hamiltonian if it visits every vertex of the graph. By the edges of a cycle we mean those edges joining two consecutive vertices of the cycle. A triangle is a complete with three vertices. A graph is triangle-free if it contains no induced triangle. Some small graphs to be referred in the sequel are depicted in Figure 1.

A universal vertex in a graph is a vertex that is adjacent to all the other vertices of the graph. An isolated vertex is a vertex that is not adjacent to any other vertex of the graph. The neighborhood of a vertex $v$ in a graph $G$ is the set $N_{G}(v)$ consisting of all the vertices that are adjacent to $v$. The degree of $v$ 

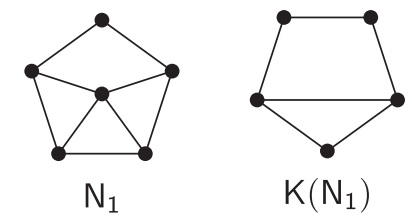

Figure 2: The graph $N_{1}$ and its clique graph

is $d_{G}(v)=\left|N_{G}(v)\right|$. The common neighborhood of an edge $e=v w$ is $N_{G}(e)=$ $N_{G}(v) \cap N_{G}(w)$, and, in general, the common neighborhood of a nonempty subset $W$ of vertices is $N_{G}(W)=\bigcap_{w \in W} N_{G}(w)$, while $N_{G}(\emptyset)=V(G)$. If $H$ is a subgraph of $G$ then $N_{H}(v)=N_{G}(v) \cap V(H), N_{H}(e)=N_{G}(e) \cap V(H)$ and $N_{H}(W)=N_{G}(W) \cap V(H)$ for every vertex $v$, every edge $e$ and every subset of vertices $W$. The closed neighborhood of $v$ is the set $N_{G}[v]=N_{G}(v) \cup\{v\}$. Two vertices $v$ and $w$ are true twins in $G$ if $N_{G}[v]=N_{G}[w]$, and false twins if $N_{G}(v)=N_{G}(w)$. The subgraph of $G$ induced by the vertex set $W \subseteq V(G)$ is denoted by $G[W]$, and $G-W$ denotes $G[V(G) \backslash W]$. A vertex set $W \subseteq V(G)$ is a vertex covering of $G$ if each edge of $G$ has at least one endpoint in $W$.

A graph $G$ is anticonnected if $\bar{G}$ is connected. An anticomponent of $G$ is the subgraph of $G$ induced by the vertices of a connected component of $\bar{G}$.

A class $\mathcal{C}$ of graphs is called hereditary if, for every graph of $\mathcal{C}$, all its induced subgraphs belong to $\mathcal{C}$. Let $G$ and $H$ be graphs. We say that $G$ is $H$-free to mean that $G$ contains no induced $H$. If $\mathcal{H}$ is a collection of graphs we say that $G$ is $\mathcal{H}$-free to mean that $G$ contains no induced $H$ for any $H \in \mathcal{H}$.

Let $\mathcal{F}$ be a family of sets. The intersection graph of $\mathcal{F}$ is a graph whose vertices are the members of $\mathcal{F}$, and such that two members of $\mathcal{F}$ are adjacent if and only if they intersect. For instance, the line graph $L(G)$ of a graph $G$ is the intersection graph of the edges of $G$. Whitney [76] proved that if $H$ and $H^{\prime}$ are connected graphs such that $L(H)=L\left(H^{\prime}\right) \neq K_{3}$ then $H=H^{\prime}$.

Another example of an intersection graph is the clique graph. The clique graph $K(G)$ of a graph $G$ is the intersection graph of the cliques of $G$. The map $K: G \mapsto K(G)$ is known as the clique graph operator or simply the clique operator. A graph $G$ is said to be $K$-perfect if $K(G)$ is perfect. If $G$ is not $K$ perfect we say that it is $K$-imperfect. Notice that the class of $K$-perfect graphs is not hereditary. For instance, the graph $N_{1}$ of Figure 2 is $K$-perfect but it contains an induced $C_{5}$ and $K\left(C_{5}\right)=C_{5}$ is imperfect. Because of this, the following terminology is introduced in [66]: a graph is hereditary $K$-perfect if all its induced subgraphs are $K$-perfect. It turns out that hereditary $K$-perfect graphs are perfect, as implied by the SPGT together with the following lemma.

Lemma 3 ([66]) A hereditary K-perfect graph has no odd holes and has no antiholes with more than 6 vertices.

Proof. It is clear that hereditary $K$-perfect graphs have no odd holes since odd holes are $K$-imperfect. All along the proof, $C_{n}$ will denote the graph such that $V\left(C_{n}\right)=\{0,1, \ldots, n-1\}$ and $E\left(C_{n}\right)=\{01,12,23, \ldots,(n-1) 0\}$. Assume that 


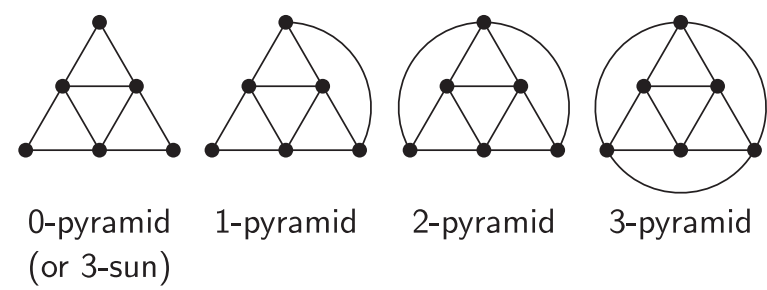

Figure 3: From left to right: 0-, 1-, 2- and 3-pyramid

$n \geq 5$ and $n \neq 6,7,9,12$. By elementary number theory, $n=5 a+3 b$ for some $a \geq 1$ and some $b \geq 0$. This implies that there exists a sequence $a_{1}, \ldots, a_{k}$ of integers taken from the set $\{2,3\}$ that satisfies the following conditions: $(i)$ $a_{1}+\cdots+a_{k}=n$; (ii) $a_{i}=2$ for some $i \in\{1, \ldots, k\}$; and (iii) for each $j=1, \ldots, k, a_{j}=2$ implies $a_{j+1}=3$ (where $a_{k+1}$ means $a_{1}$ ). Assume such a sequence $\left\{a_{i}\right\}$ is given and define $b_{i}$ equal to $a_{1}+\cdots+a_{i}$ modulo $n$ for each $i=1, \ldots, k$. In particular, $b_{k}=0$. Let $Q_{1}=\left\{b_{1}, b_{2}, \ldots, b_{k}\right\}, Q_{2}=Q_{1}+2$, $Q_{3}=Q_{1}+4, Q_{4}=Q_{1}+1$, and $Q_{5}=Q_{1}+3$, where $A+p=\{a+p: a \in A\}$ and the sum is taken modulo $n$. Then, $Q_{i}$ is a clique of $\overline{C_{n}}$ for $i=1,2, \ldots, 5$ and, by construction, $Q_{1} Q_{2} \ldots Q_{5}$ is an odd hole in $K\left(\overline{C_{n}}\right)$. Finally, observe that $K\left(\overline{C_{7}}\right)=\overline{C_{7}}$; that if $Q_{1}=\{0,2,4,6\}$ then $\left\{Q_{1}, Q_{1}+1, Q_{1}+2, \ldots, Q_{1}+8\right\}$ induces a $\overline{C_{9}}$ in $K\left(\overline{C_{9}}\right)$; and that if $Q_{1}=\{0,2,5,7,9\}$ and $Q_{2}=\{1,3,5,7,10\}$ then $\left\{Q_{1}, Q_{1}+1, Q_{1}+2, Q_{1}+3, Q_{1}+9, Q_{2}, Q_{2}+1, Q_{2}+2, Q_{2}+3\right\}$ induces a $\overline{C_{9}}$ in $K\left(\overline{C_{12}}\right)$.

Interestingly, hereditary $K$-perfection has been implicitly characterized when restricted to several graph classes; many of these characterizations are presented in Section 5.

A family $\mathcal{F}$ of nonempty sets is said to satisfy the Helly property if every nonempty subfamily of $\mathcal{F}$ of pairwise intersecting members has nonempty intersection. A graph $G$ is said to be clique-Helly $(\mathrm{CH})$ if the family of its cliques satisfies the Helly property. The graphs of Figure 3 are examples of graphs that are not clique-Helly. Clique-Helly graphs were characterized in [32] and independently in [71]. Notice that any graph with a universal vertex is clique-Helly and thus a clique-Helly graph may contain any prescribed induced subgraph. Instead, a graph is hereditary clique-Helly (HCH) [64] if all its induced subgraphs are clique-Helly. Prisner gave several characterizations of hereditary clique-Helly graphs, one by means of minimal forbidden induced subgraphs:

Theorem 4 ([64]) A graph is hereditary clique-Helly if and only if it contains none of the graphs of Figure 3 as induced subgraph.

In the sequel, we call any of the graphs in Figure 3 a pyramid. The graph 0 -pyramid is also called 3 -sun. In [64] and [74], it is proved that a hereditary clique-Helly graph $G$ has at most $|V(G)|+|E(G)|$ cliques.

Let $G$ and $H$ be two graphs. Assume that $V(G) \cap V(H)=\emptyset$. The disjoint 
union of $G$ and $H$ is a graph $G \cup H$ whose vertex set is $V(G) \cup V(H)$ and whose edge set is $E(G) \cup E(H)$. The disjoint union is clearly an associative operation, and for each nonnegative integer $t$ we will denote by $t G$ the disjoint union of $t$ copies of $G$. The join of $G$ and $H$ is a graph $G+H$ whose vertex set is $V(G) \cup V(H)$ and whose edge set is $E(G) \cup E(H) \cup\{v w: v \in V(G), w \in V(H)\}$.

A graph is bipartite if its vertex set can be partitioned into two (possibly empty) stable sets. A graph is chordal if every cycle of length at least 4 has at least one chord. A comparability graph is a graph that admits a transitive acyclic orientation of its edges. Bipartite and chordal graphs can be recognized in linear time and comparability graphs can be recognized in polynomial time [65, 70]. Bipartite, chordal and comparability graphs are subclasses of perfect graphs.

A cograph $[29]$ is a $P_{4}$-free graph, that is, a graph without chordless paths on 4 vertices. Equivalently, cographs are those graphs that can be obtained from isolated vertices by successively applying disjoint union and join operations. Cographs form a well-known class of perfect graphs.

We will study in this survey two superclasses of cographs: $P_{4}$-tidy graphs and tree-cographs. A graph $G=(V, E)$ is $P_{4}$-tidy if for every vertex set $A$ inducing a $P_{4}$ in $G$ there is at most one vertex $v \in V \backslash A$ such that $G[A \cup\{v\}]$ contains at least two induced $P_{4}$ 's. They were introduced in [45]. A starfish is a graph whose vertex set can be partitioned into three sets $S, C$ and $R$, where each of the following conditions holds: (1) $S=\left\{s_{1}, \ldots, s_{t}\right\}$ is a stable set and $C=\left\{c_{1}, \ldots, c_{t}\right\}$ is a clique, for some $t \geq 2 ;(2) s_{i}$ is adjacent to $c_{j}$ if and only if $i=j$; and (3) $R$ is allowed to be empty and if it is not, then all the vertices in $R$ are adjacent to all the vertices in $C$ and nonadjacent to all the vertices in $S$. An urchin is a graph whose vertex set can be partitioned into three sets $S, C$, and $R$ satisfying the same conditions (1) and (3) but that instead of condition (2) satisfies: (2') $s_{i}$ is adjacent to $c_{j}$ if and only if $i=j$. Clearly, urchins are the complements of starfishes and vice versa. A fat starfish (resp. fat urchin) arises from a starfish (resp. urchin) with partition $(S, C, R)$ by substituting exactly one vertex of $S \cup C$ by $K_{2}$ or $2 K_{1}$.

Theorem 5 ([45]) If $G$ is a $P_{4}$-tidy graph, then exactly one of the following statements holds:

1. $G$ or $\bar{G}$ is disconnected;

2. $G$ is isomorphic to $C_{5}, P_{5}, \overline{P_{5}}$, a starfish, a fat starfish, an urchin, or a fat urchin.

Tree-cographs were defined in [72] by the following recursive definition:

1. Every tree is a tree-cograph.

2. If $G$ is a tree-cograph, then $\bar{G}$ is a tree-cograph.

3. The disjoint union of tree-cographs is a tree-cograph.

This definition implies that if $G$ is a tree-cograph, then either $G$ or $\bar{G}$ is disconnected, or $G$ is a tree or the complement of a tree. Tree-cographs are also a subclass of perfect graphs. 
Distance-hereditary graphs form another superclass of cographs. A graph $G$ is called distance-hereditary if and only if the distance between any two vertices of $G$ is the same in every connected induced subgraph of $G$ containing the two vertices. Equivalently, a graph is distance-hereditary if and only if it is \{house,domino,gem\}-free and has no holes of length at least 5 [3].

A circular-arc graph [46] is the intersection graph of arcs of the unit circle. A representation of a circular-arc graph is a collection of arcs (of the unit circle), each corresponding to a unique vertex of the graph, such that two arcs intersect if and only if the corresponding vertices are adjacent. A Helly circular-arc (HCA) graph [44] is a circular-arc graph admitting a representation whose arcs satisfy the Helly property.

Let $G$ be a graph, $Q_{1}, \ldots, Q_{k}$ all its cliques and $v_{1}, \ldots, v_{n}$ all its vertices. A clique matrix (or clique-vertex incidence matrix) of $G$ is the $k \times n$ matrix $A=\left(a_{i j}\right)$ where $a_{i j}$ is 1 if $v_{j} \in Q_{i}$ and 0 otherwise. The clique matrix of a graph is unique up to permutations of rows and/or columns. Let $A$ be a $m \times n$ zero-one matrix. We say that $A$ is perfect if the set packing polytope

$$
\left\{x \in \mathbb{R}^{n} \mid x \geq \mathbf{0}, A x \leq \mathbf{1}\right\}
$$

has all integer extreme points. Perfect graphs and perfect matrices are related by the following result [26, 63]: a graph is perfect if and only if its clique matrix is perfect.

A zero-one matrix $A$ is said to be balanced if and only if it contains no odd square submatrix with exactly two 1's in each row and in each column. Clearly, balancedness is preserved by row permutations, column permutations and transpositions. There is a forbidden submatrix characterization for balanced matrices in terms of perfect matrices: Let $A$ be a zero-one matrix. Then $A$ is balanced if and only if all submatrices of $A$ are perfect $[4,63]$. In particular, balanced matrices are perfect. By analogy with the relation between perfect graphs and perfect matrices, Dahlhaus, Manuel and Miller proposed to call a graph balanced if its clique matrix is balanced [30]. There is a characterization of balanced graphs in terms of forbidden structures defined as follows. An unbalanced cycle of a graph $G$ is an odd cycle $C$ such that for each edge $e$ of $C$ there exists a (possibly empty) complete $W_{e}$ of $G$ such that $W_{e} \subseteq N_{G}(e) \backslash C$ and $N_{C}\left(W_{e}\right) \cap N_{C}(e)=\emptyset$.

Theorem $6([4,14])$ A graph is balanced if and only if it contains no unbalanced cycle.

In [64], Prisner proved that a graph is hereditary clique-Helly if and only if its clique matrix does not contain a $3 \times 3$ submatrix with exactly two 1 's in each row and in each column. In particular, it turns out that balanced graphs are hereditary clique-Helly, thus the number of cliques of a balanced graph is bounded by its number of vertices plus its number of edges. Dahlhaus, Manuel and Miller observed that combining the polynomial-time algorithm in [73] that outputs the clique matrix of a hereditary clique-Helly graph, together with the polynomialtime algorithm in [27] that recognizes balanced matrices, a polynomial-time 


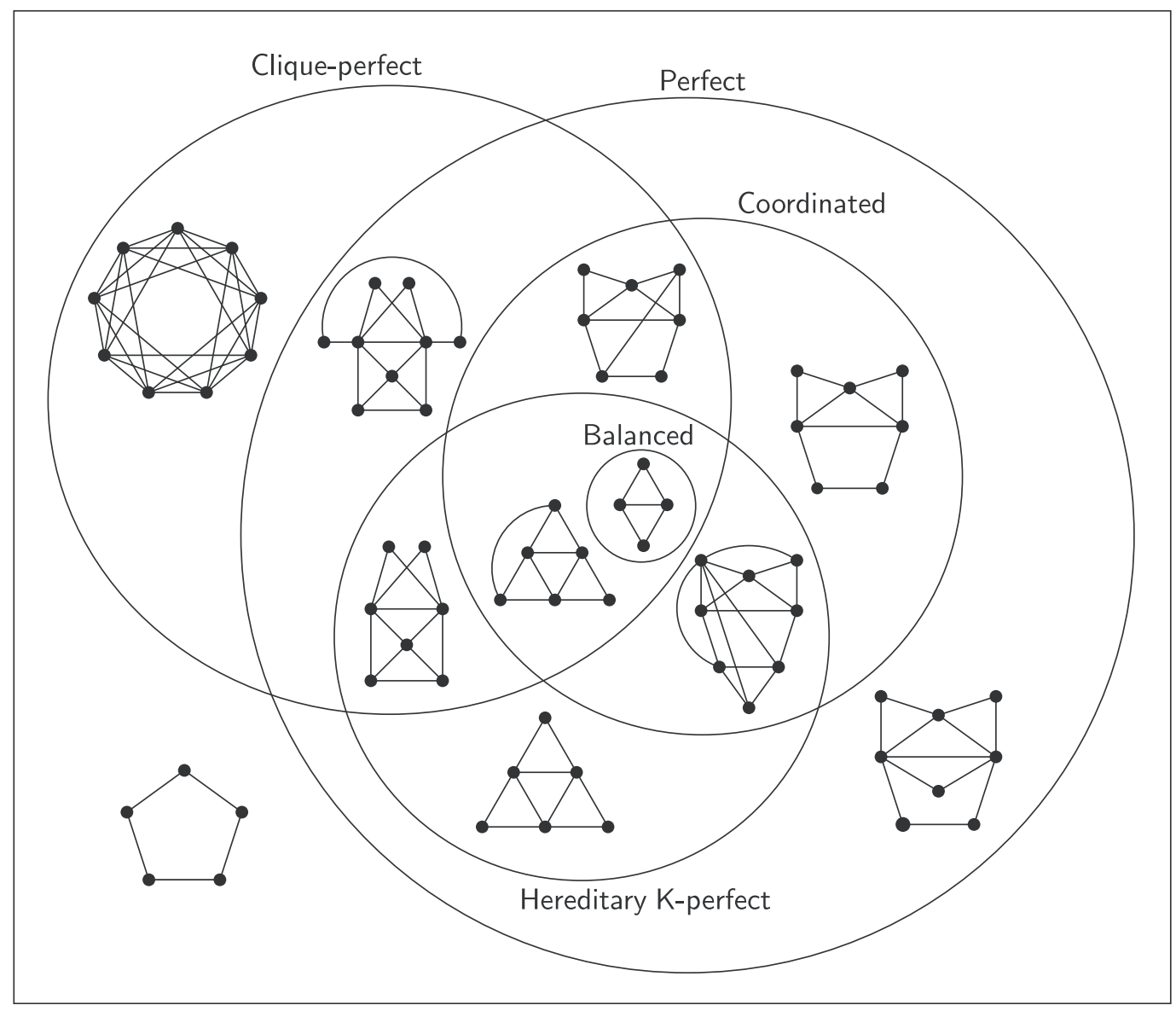

Figure 4: Inclusions and intersections of the studied classes related to coordinated graphs, together with separating examples

algorithm to recognize balanced graphs is obtained. The algorithm proposed in [27] by Conforti, Cornuéjols, Kapoor and Vušković was quite involved, but Zambelli [77] developed a simpler polynomial-time algorithm to test balancedness of a matrix.

Figures 4 and 5 show the inclusion and intersection schemes of the graph classes that are the subject of this paper related to coordinated and neighborhoodperfect graphs, respectively. 


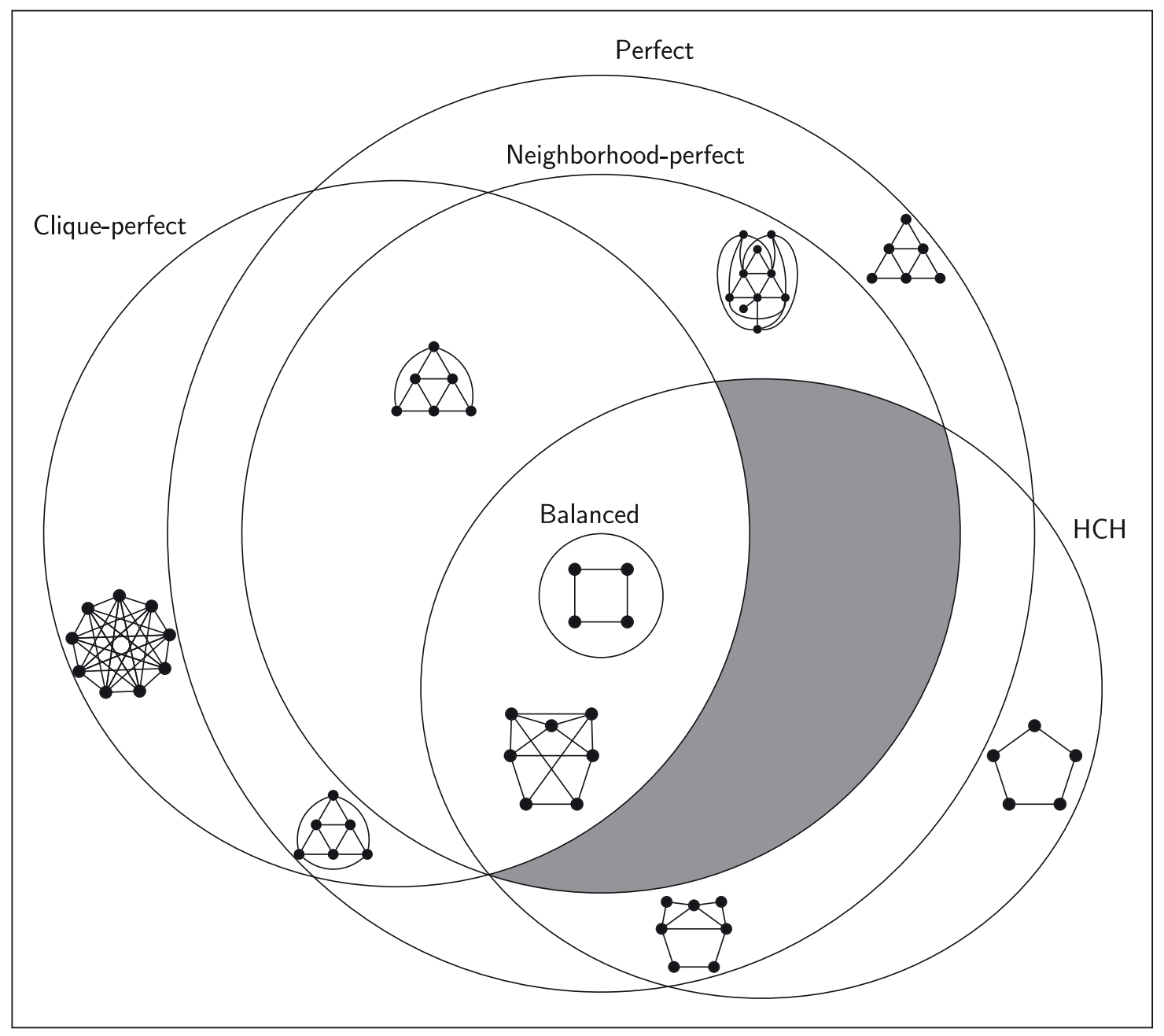

Figure 5: Inclusions and intersections of the studied classes related to neighborhood-perfect graphs, with separating examples. The shaded region corresponds to an empty set. 


\section{Connection with hypergraph theory}

A hypergraph $H$ is an ordered pair $(X, \mathcal{E})$ where $X$ is a finite set and $\mathcal{E}$ is a family of nonempty subsets of $X$. The elements of $X$ are the vertices of $H$ and the elements of $\mathcal{E}$ are the hyperedges of $H$. If $x_{1}, \ldots, x_{n}$ are the vertices of $H$ and $E_{1}, \ldots, E_{m}$ are the hyperedges of $H$ then a hyperedge-vertex incidence matrix of $H$ is the $m \times n$ matrix $A=\left(a_{i j}\right)$ where $a_{i j}$ is 1 if $x_{j} \in E_{i}$ and 0 otherwise. A hypergraph has the Helly property if every nonempty family of pairwise intersecting hyperedges has a nonempty intersection. The line graph (or representative graph) of a hypergraph $H$, denoted by $L(H)$, is the intersection graph of the family $\mathcal{E}$ of hyperedges of $H$.

We will restrict ourselves to hypergraphs $(X, \mathcal{E})$ where $\bigcup \mathcal{E}=X$. A partial hypergraph of $H$ is a hypergraph $H^{\prime}$ whose hyperedge set $\mathcal{E}^{\prime}$ is a subset of the hyperedge set of $H$ and whose vertex set is the union of the members of $\mathcal{E}^{\prime}$.

We will be mostly interested in studying clique hypergraphs of graphs. Namely, the clique hypergraph of a graph $G$ is the hypergraph $\mathcal{K}(G)=(X, \mathcal{E})$ where $X$ is the set of vertices of $G$ and $\mathcal{E}$ is the family of cliques of $G$. The hyperedge-vertex incidence matrix of $\mathcal{K}(G)$ is the clique matrix of $G$, and $G$ is clique-Helly if and only if $\mathcal{K}(G)$ has the Helly property. Besides, clique graph and clique hypergraph are related in the following way: $K(G)=L(\mathcal{K}(G))$.

\subsection{The König property}

A matching of $H$ is a family of pairwise disjoint hyperedges and the matching number $\nu(H)$ is the maximum cardinality of a matching of $H$. A transversal of $H$ is a set of vertices that meet all the hyperedges and the transversal number $\tau(H)$ is the minimum cardinality of a transversal of $H$. Clearly, $\nu(H) \leq \tau(H)$ for each hypergraph $H$. A hypergraph is said to satisfy the König property if $\nu(H)=\tau(H)$. Notice that $\alpha_{\mathrm{c}}(G)=\nu(\mathcal{K}(G))$ and $\tau_{\mathrm{c}}(G)=\tau(\mathcal{K}(G))$. Thus, we have by definition:

Remark 1 A graph $G$ is clique-perfect if and only if the clique hypergraph $\mathcal{K}\left(G^{\prime}\right)$ has the König property for each induced subgraph $G^{\prime}$ of $G$.

\subsection{The colored edge property}

Another property of hypergraphs being of our interest is the following. Let $H$ be a hypergraph. The chromatic index $\gamma(H)$ of $H$ is the least number of colors necessary to color the hyperedges of $H$ such that any two intersecting hyperedges are colored with different colors. The degree $d_{H}(x)$ of a vertex $x$ of $H$ is the number of hyperedges of $H$ containing $x$. The maximum degree of the hypergraph $H$ is defined as $\Delta(H)=\max _{x \in X} d_{H}(x)$. Clearly, $\Delta(H) \leq$ $\gamma(H)$ for any hypergraph $H$. Finally, a graph is said to have the colored edge property [6, p. 15] if and only if $\gamma(H)=\Delta(H)$. Since $\gamma_{\mathrm{c}}(G)=\gamma(\mathcal{K}(G))$ and $\Delta_{\mathrm{c}}(G)=\Delta(\mathcal{K}(G))$ hold, we obtain: 
Remark 2 A graph $G$ is coordinated if and only if the clique hypergraph $\mathcal{K}\left(G^{\prime}\right)$ has the colored edge property for each induced subgraph $G^{\prime}$ of $G$.

\subsection{Normality}

Lovász proved the following (we use the formulation of Berge [6, pp. 195-197]):

Theorem 7 ([56]) Let $H$ be a hypergraph, $A_{H}$ be the hyperedge-vertex incidence matrix of $H$ and $A_{H}^{T}$ be its transpose. Then the following conditions are equivalent:

1. Every partial hypergraph of $H$ has the Konnig property.

2. Every partial hypergraph of $H$ has the colored edge property.

3. The matrix $A_{H}^{T}$ is perfect.

4. $H$ satisfies the Helly property and $L(H)$ is perfect.

Any hypergraph satisfying any of these conditions is said to be normal. Since $K(G)=L(\mathcal{K}(G))$, it follows as a corollary:

Corollary 8 Let $G$ be a graph, $A_{G}$ the clique matrix of $G$ and $A_{G}^{T}$ be its transpose. Then the following are equivalent:

1. Every partial hypergraph of $\mathcal{K}(G)$ has the König property.

2. Every partial hypergraph of $\mathcal{K}(G)$ has the colored edge property.

3. The matrix $A_{G}^{T}$ is perfect.

4. $G$ is clique-Helly and $K(G)$ is perfect.

In light of this theorem we introduce the following terminology: a graph $G$ will be called clique-normal if $G$ is clique-Helly and $K(G)$ is perfect, or equivalently, if its clique hypergraph $\mathcal{K}(G)$ is normal. Notice that an induced subgraph of a clique-normal graph may not be clique-normal. For instance, the graph $N_{1}$ of Figure 2 is clique-normal but contains an induced $C_{5}$ which is not even $K$-perfect.

So we introduce also the following definition: a graph $G$ is said to be hereditary clique-normal if all the induced subgraphs of $G$ are clique-normal. Equivalently, $G$ is hereditary clique-normal if it is hereditary clique-Helly and hereditary $K$-perfect. Combining Corollary 8 with Remarks 1 and 2 it follows:

Corollary 9 If $G$ is hereditary clique-normal (i.e., hereditary clique-Helly and hereditary $K$-perfect) then $G$ is clique-perfect and coordinated.

The converse is not true because there are graphs that are clique-perfect and coordinated but not even hereditary $K$-perfect (see Figure 4 ). Nevertheless, Corollaries 16 and 20 of Section 4 can be regarded as partial converses of Corollary 9.

A different characterization of clique-normal graphs arises from defining clique subgraphs, which are closer to partial hypergraphs of the clique hypergraph than induced subgraphs. Let $G$ be a graph, $\mathcal{Q}$ the set of cliques of $G$ and 
$\mathcal{Q}^{\prime} \subseteq \mathcal{Q}$. Denote by $G_{\mathcal{Q}^{\prime}}$ the subgraph of $G$ formed exactly by the vertices and edges corresponding to the cliques in $\mathcal{Q}^{\prime}$. If every clique of $G_{\mathcal{Q}^{\prime}}$ is also a clique of $G$ then $G_{\mathcal{Q}^{\prime}}$ is called a clique subgraph of $G$ [13]. A graph $G$ is called $c$-cliqueperfect if $\tau_{\mathrm{c}}(H)=\alpha_{\mathrm{c}}(H)$ for every clique subgraph $H$ of $G$, and $c$-coordinated if $\gamma_{\mathrm{c}}(H)=\Delta_{\mathrm{c}}(H)$ for every clique subgraph $H$ of $G$.

By definition, if $H$ is a clique subgraph of a graph $G$ then $K(H)$ is an induced subgraph of $K(G)$. This property allows to prove the following theorem:

Theorem $10([\mathbf{1 2}, \mathbf{1 3}])$ Let $G$ be a clique-normal graph. Then $G$ is c-cliqueperfect and $c$-coordinated.

Moreover, when $G$ is hereditary clique-Helly, every induced subgraph of $K(G)$ is the clique graph of a clique subgraph of $G$ [64]. So the following holds:

Theorem $11([\mathbf{1 2}, \mathbf{1 3}])$ Let $G$ be a hereditary clique-Helly graph. Then the following statements are equivalent:

1. $K(G)$ is perfect.

2. $G$ is c-clique-perfect.

3. $G$ is c-coordinated.

4. $G$ is clique-normal.

It remains an open question whether the equivalence among assertions (1), (2), and (3) of the above theorem holds for (general) clique-Helly graphs $G$.

Berge defined in 1969 a hypergraph to be balanced (cf. [33, p. 397]) if its hyperedge-vertex incidence matrix is balanced. Recall that balanced graphs are those whose clique matrix is balanced, that is, those graphs whose clique hypergraph is balanced. We have also the following:

Theorem $12([7,56])$ If a hypergraph is balanced then it is also normal.

Since the class of balanced graphs is hereditary $[14,46]$ then:

Corollary 13 Balanced graphs are hereditary clique-normal (i.e., hereditary clique-Helly and hereditary K-perfect). In particular, balanced graphs are cliqueperfect and coordinated.

The class of balanced graphs is a common subclass of clique-perfect and coordinated graphs that is interesting from a computational point of view. In fact, the problems of determining each of the parameters $\alpha_{\mathrm{c}}, \tau_{\mathrm{c}}, \Delta_{\mathrm{c}}$ and $\gamma_{\mathrm{c}}$ are NP-complete [20], NP-hard [38], \#P-complete [8] and $\{\# \mathrm{P}, \mathrm{NP}\}$-hard [8], respectively. However, all these problems are known to be polynomially solvable when restricted to balanced graphs. Indeed, as we already mentioned, the size of the clique matrix of a balanced graph is bounded by a polynomial in the number of vertices and, consequently, can be computed in polynomial time. This, combined with the fact that the set packing and set covering polyhedra of balanced matrices are integral [41], implies the following:

Theorem $14([30,41])$ Each of the parameters $\alpha_{\mathrm{c}}, \tau_{\mathrm{c}}, \Delta_{\mathrm{c}}$ and $\gamma_{\mathrm{c}}$ can be computed in polynomial time (in the number of vertices) for balanced graphs. 


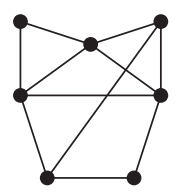

Figure 6: An example of an $\alpha(K)$-perfect and $\chi(K)$-perfect but $K$-imperfect graph

\section{Connection with the clique graph operator}

The following result relates the parameters used to define clique-perfect graphs with the parameters used to define perfect graphs applied to the clique graph:

Theorem 15 ([13]) Let $G$ be a graph. Then:

1. $\alpha_{\mathrm{c}}(G)=\alpha(K(G))$.

2. $\tau_{\mathrm{c}}(G) \geq \theta(K(G))$.

3. If $G$ is clique-Helly then $\tau_{\mathrm{c}}(G)=\theta(K(G))$.

The theorem above asserts, in particular, that

$$
\alpha_{\mathrm{c}}(G)=\alpha(K(G)) \leq \theta(K(G)) \leq \tau_{\mathrm{c}}(G) \text { holds for any graph } G
$$

and we shall notice that a graph is clique-perfect exactly when both inequalities are satisfied at equality for each of its induced subgraphs.

A graph $G$ is called $\alpha$-good when $\alpha(G)=\theta(G)$, and $\alpha$-bad otherwise. Contrary to the case of perfect graphs, the equality is not imposed to the induced subgraphs, and $\alpha$-goodness is strictly weaker than perfection. We will say that a graph $G$ is $\alpha(K)$-good if $K(G)$ is $\alpha$-good, i.e. if $\alpha(K(G))=\theta(K(G))$. We will say that it is $\alpha(K)$-bad otherwise. Finally, we define a graph to be $\alpha(K)$-perfect if each of its induced subgraphs is $\alpha(K)$-good, or equivalently, if $\alpha\left(K\left(G^{\prime}\right)\right)=\theta\left(K\left(G^{\prime}\right)\right)$ for each induced subgraph $G^{\prime}$ of $G$. The graph depicted in Figure 6 is an example of an $\alpha(K)$-perfect graph that is not hereditary $K$-perfect. Notice that given a graph $G$, the clique graph $K(G)$ may contain some induced subgraphs which are not clique graphs of any induced subgraph of $G$ and this is one reason why $\alpha(K)$-perfection turns out to be strictly weaker property than hereditary $K$-perfection.

With all this terminology, Theorem 15 implies the following variant of Corollary 9:

Corollary 16 ([13]) If $G$ is a clique-perfect graph then $G$ is $\alpha(K)$-perfect. Furthermore, if $G$ is hereditary clique-Helly then the converse also holds.

Thus the class of $\alpha(K)$-perfect graphs is a superclass of both clique-perfect graphs and hereditary $K$-perfect graphs. Below we introduce some graphs that are known to be clique-imperfect and study whether they are $\alpha(K)$-good or not. 
An $r$-sun or simply sun [39] is a chordal graph $G$ on $2 r$ vertices, $r \geq 3$, whose vertex set can be partitioned into two sets, $W=\left\{w_{1}, \ldots, w_{r}\right\}$ and $U=$ $\left\{u_{1}, \ldots, u_{r}\right\}$, such that $W$ is a stable set and for each $i$ and $j, w_{j}$ is adjacent to $u_{i}$ if and only if $i=j$ or $i \equiv j+1 \bmod r$. A sun is odd if $r$ is odd. A sun is complete if $U$ is a complete.

The concept of suns was later extended as follows. Let $G$ be a graph and $C$ be a cycle of $G$ not necessarily induced. An edge of $C$ is non-proper (or improper) if it forms a triangle with some vertex of $C$. An $r$-generalized sun, $r \geq 3$, is a graph $G$ whose vertex set can be partitioned into two sets: a cycle $C$ of $r$ vertices, with all its non-proper edges $\left\{e_{j}\right\}_{j \in J}$ ( $J$ is permitted be an empty set) and a stable set $U=\left\{u_{j}\right\}_{j \in J}$, such that for each $j \in J, u_{j}$ is adjacent exactly to the endpoints of $e_{j}$. An $r$-generalized sun is said to be odd if $r$ is odd. Clearly odd holes and odd suns are odd generalized suns. We call a cycle proper if none of its edges is improper. By definition, proper odd cycles are odd generalized suns. It turns out that odd generalized suns are not clique-perfect. Indeed, the following holds.

Theorem 17 ([13, 36]) Odd generalized suns and antiholes of a length not divisible by 3 are clique-imperfect.

It is not hard to see that the 3 -sun is hereditary $K$-perfect and, in particular, $\alpha(K)$-perfect. We will now show that this is the only odd-generalized sun that is $\alpha(K)$-good. More precisely:

Theorem 18 Odd generalized suns different from the 3-sun and antiholes of a length not divisible by 3 are $\alpha(K)$-bad.

Proof. Let $G$ be a $(2 r+1)$-generalized sun for some $r \geq 2$ and let $C,\left\{e_{j}\right\}_{j \in J}$ and $\left\{u_{j}\right\}_{j \in J}$ be as in the definition of odd generalized suns. In [13] it is proved that $\alpha(K(G))=\alpha_{\mathrm{c}}(G) \leq r$. We claim that $\theta(K(G)) \geq r+1$. Notice that $\theta(K(G))$ is the minimum number of colors needed to color the cliques of $G$ in such a way that if two cliques receive the same color then they intersect. Consider any such coloring with $\theta(K(G))$ colors. We assign to each edge $e=x y$ of $C$ a clique $Q_{e}$ of $G$ as follows: if $e$ is proper then $Q_{e}=\{x, y\}$ and if $e$ is improper then there exists $j \in J$ such that $e=e_{j}$ and let $Q_{e}=\left\{x, y, u_{j}\right\}$. Clearly, $Q_{e} \cap Q_{e^{\prime}} \neq \emptyset$ if and only if $e^{\prime}$ and $e$ are adjacent. Since $r \geq 2$ then the edges of $C$ do not form triangles and then there are not three pairwise different and pairwise adjacent edges of $C$. This means that among all the cliques $Q_{e}$ there are no more than two of them that receive the same color, and therefore $\theta(K(G)) \geq(2 r+1) / 2>r \geq \alpha(K(G))$.

Regarding antiholes, let $n \geq 5$ and $n$ not be a multiple of 3 . Then $\alpha\left(K\left(\overline{C_{n}}\right)\right)=$ 2 [36]. By the proof of Lemma $3, K\left(\overline{C_{n}}\right)$ is not the complement of a bipartite graph, and this accounts for $\theta\left(K\left(\overline{C_{n}}\right)\right)>2$. (In fact, it is easy to see that $\theta\left(K\left(\overline{C_{n}}\right)\right)=3$ because given three consecutive vertices $v_{1}, v_{2}, v_{3}$ of $C_{n}$, the cliques of $\overline{C_{n}}$ that contain $v_{i}$ constitute a clique of $K\left(\overline{C_{n}}\right)$, and conversely every clique of $K\left(\overline{C_{n}}\right)$ contains at least one vertex from $\left\{v_{1}, v_{2}, v_{3}\right\}$.) 
In particular, $\overline{C_{8}}$ is not $\alpha(K)$-good, which shows that perfect graphs are not necessarily $\alpha(K)$-perfect. Also notice that antiholes $\overline{C_{3 k}}$ are $\alpha(K)$-perfect because they are clique-perfect. This shows that $\alpha(K)$-perfect graphs are not necessarily perfect. We have the following:

$$
\text { clique-perfect } \subset \alpha(K) \text {-perfect } \subset\{(2 r+1) \text {-generalized sun, } r>1\} \text {-free } \cap
$$

$$
\left\{\overline{C_{n}}: n \geq 5, n \neq 3 k\right\} \text {-free }
$$

To see that the inclusions are proper, notice that the 3 -sun is $\alpha(K)$-perfect (because it is hereditary $K$-perfect) but not clique-perfect because $\alpha_{\mathrm{c}}(3$-sun $)=1$ and $\tau_{\mathrm{c}}(3$-sun $)=2$, and that $S_{2}$ (cf. Figure 10, p. 28) contains no induced odd generalized sun and has no antiholes but is not $\alpha(K)$-good since $\alpha\left(K\left(S_{2}\right)\right)=2$ and $\theta\left(K\left(S_{2}\right)\right)=3$.

According to equation $(*)$ (p. 15), we propose to classify the minimal forbidden induced subgraphs for clique-perfection into two classes (whenever we say minimal, it should be understood in the sense of induced subgraphs). The class $\mathcal{O}_{1}$ of those graphs $G$ with $\alpha(K(G))<\theta(K(G))$, and the class $\mathcal{O}_{2}$ of those graphs $G$ for which $\theta(K(G))<\tau_{\mathrm{c}}(G)$ holds. We will call its elements simply obstructions. Notice that all odd generalized suns belong to $\mathcal{O}_{1}$ with the only exception of the 3 -sun that consequently belongs to $\mathcal{O}_{2}$. If $n \geq 5$ and $n$ is not a multiple of 3 then also $\overline{C_{n}} \in \mathcal{O}_{1}$. The classes $\mathcal{O}_{1}$ and $\mathcal{O}_{2}$ may actually overlap. Indeed there are graphs $G$ for which $\alpha(K(G))<\theta(K(G))<\tau_{\mathrm{c}}(G)$, but all examples of such graphs $G$ that we know contain an induced $C_{5}$ and so they are not minimally clique-imperfect.

Due to Theorem 15 all graphs $G$ that are hereditary clique-Helly satisfy $\theta(K(G))=\tau_{\mathrm{c}}(G)$, thus, if an obstruction contains no pyramid then it belongs to $\mathcal{O}_{1}$. It would be interesting to find a weaker condition on the obstructions $G$ that still ensures that $\theta(K(G))=\tau_{\mathrm{c}}(G)$ holds. We wonder, for instance, the following:

Problem 1 Is there any minimally clique-imperfect graph $G$ that contains no induced 0-, 1-, or 2-pyramid and such that $\theta(K(G))=\tau_{\mathrm{c}}(G)$ does not hold?

Clearly, if there is any such graph $G$ then it must contain an induced 3pyramid. Currently, we do not know any example to answer this question affirmatively.

In Table 1 the number of minimally clique-imperfect graphs in the classes $\mathcal{O}_{1}$ and $\mathcal{O}_{2}$ were tabulated by computer, and suggest that the class $\mathcal{O}_{1}$ is much larger than the class $\mathcal{O}_{2}$.

The analogous to Theorem 15 for coordinated graphs was formulated in [12]:

Theorem 19 ([12]) Let $G$ be a graph. Then:

1. $\gamma_{\mathrm{c}}(G)=\chi(K(G))$.

2. $\Delta_{\mathrm{c}}(G) \leq \omega(K(G))$.

3. If $G$ is clique-Helly then $\Delta_{c}(G)=\omega(K(G))$. 


\begin{tabular}{cccc}
\hline $\begin{array}{c}\text { Number } \\
\text { of vertices }\end{array}$ & $\begin{array}{c}\text { Minimally clique-imperfect } \\
\text { graphs (those that in addition } \\
\text { are odd generalized suns) }\end{array}$ & $\begin{array}{c}\text { Elements } \\
\text { of } \mathcal{O}_{1}\end{array}$ & $\begin{array}{c}\text { Elements } \\
\text { of } \mathcal{O}_{2}\end{array}$ \\
\hline$\leq 4$ & $0(0)$ & 0 & 0 \\
5 & $1(1)$ & 1 & 0 \\
6 & $1(1)$ & 0 & 1 \\
7 & $3(2)$ & 3 & 0 \\
8 & $13(2)$ & 7 & 6 \\
9 & $45(5)$ & 31 & 14 \\
10 & $201(10)$ & 158 & 43 \\
11 & $1048(21)$ & 875 & 173 \\
\hline
\end{tabular}

Table 1: Classifying minimally clique-imperfect graphs

As a consequence,

$$
\Delta_{\mathrm{c}}(G) \leq \omega(K(G)) \leq \chi(K(G))=\gamma_{\mathrm{c}}(G) \text { holds for any graph } G,
$$

and coordinated graphs are exactly those for which both inequalities are satisfied at equality for each induced subgraph. Again, as a corollary of Theorem 19 we will obtain Corollary 20, which is another variant of Corollary 9 but in terms of $\chi$-goodness. A graph is called $\chi$-good if $\chi(G)=\omega(G)$. The same property is not required for induced subgraphs, and for this reason $\chi$-goodness is strictly weaker than perfection. Let us call a graph $\chi(K)$-good if its clique graph is $\chi$ good, and define a graph $G$ to be $\chi(K)$-perfect if all its induced subgraphs are $\chi(K)$-good. Equivalently, $G$ is $\chi(K)$-perfect if and only if $\chi\left(K\left(G^{\prime}\right)\right)=\omega\left(K\left(G^{\prime}\right)\right)$ for each induced subgraph $G^{\prime}$ of $G$. Again, the graph of Figure 6 shows that $\chi(K)$-perfection is strictly weaker than hereditary $K$-perfection. Finally, the analogous to Corollary 16 is the following.

Corollary 20 If $G$ is a coordinated graph then $G$ is $\chi(K)$-perfect. Furthermore, if $G$ is hereditary clique-Helly then the converse also holds.

Therefore $\chi(K)$-perfect graphs constitute a superclass of both coordinated graphs and hereditary $K$-perfect graphs. In [12] the following is proved for coordinated graphs:

Theorem 21 ([12]) If $G$ is a coordinated graph then $G$ has no odd holes and has no antiholes with more than 6 vertices.

The analogous for hereditary $K$-perfect graphs was proved in Lemma 3. In the next theorem we extend both results to the class of $\chi(K)$-perfect graphs. 
Theorem 22 If $G$ is $\chi(K)$-perfect then $G$ has no odd holes and has no antiholes with more than 6 vertices.

Proof. Since $K\left(C_{n}\right)=C_{n}$ then odd holes are not $\chi(K)$-good. In [12] it is shown that $\chi\left(K\left(\overline{C_{n}}\right)\right) \geq\left(\left|V\left(K\left(\overline{C_{n}}\right)\right)\right|-1\right) / 2$ for each $n \geq 5$ and a recursive formula is given for $\left|V\left(K\left(\overline{C_{n}}\right)\right)\right|$ which implies that $\left|V\left(K\left(\overline{C_{n}}\right)\right)\right|>2 n+1$ for $n \geq 8$. All along the proof, $C_{n}$ will denote the graph such that $V\left(C_{n}\right)=\{0,1, \ldots, n-1\}$ and $E\left(C_{n}\right)=\{01,12,23, \ldots,(n-1) 0\}$. Let $Q$ be a clique of $\overline{C_{n}}$ and define the orbit of $Q$ as $O(Q)=\{Q+j: 0 \leq j \leq n-1\}$ where $Q+j$ stands for $\{a+j: a \in Q\}$ and the sum is taken modulo $n$. The period of $Q$ is $|O(Q)|$. The orbits induce a partition of the cliques of $\overline{C_{n}}$ and the period of each clique $Q$ divides $n$. For each clique $Q$ of $\overline{C_{n}}$ it holds that $(Q+j) \cap(Q+j+1)=\emptyset$ and consequently any complete of $K\left(\overline{C_{n}}\right)$ cannot contain both $Q+j$ and $Q+j+1$ for any $j$. Therefore, each complete of $K\left(\overline{C_{n}}\right)$ contains at most $\lfloor|O(Q)| / 2\rfloor$ members of $O(Q)$, for each clique $Q$ of $\overline{C_{n}}$ (where $\lfloor x\rfloor$ denotes the largest integer not exceeding $x$ ).

Since $K\left(\overline{C_{7}}\right)=\overline{C_{7}}$ then $\overline{C_{7}}$ is not $\chi(K)$-good, so henceforth we can assume that $n \geq 8$. We claim that there is a set $S$ that is the union of orbits of cliques of $\overline{C_{n}}$ such that any complete of $K\left(\overline{C_{n}}\right)$ contains at most $|S| / 2-1$ elements of $S$. This will imply that $\omega\left(K\left(\overline{C_{n}}\right)\right) \leq\left(\left|V\left(K\left(\overline{C_{n}}\right)\right)\right|-2\right) / 2$, which together with $\chi\left(K\left(\overline{C_{n}}\right)\right) \geq\left(\left|V\left(K\left(\overline{C_{n}}\right)\right)\right|-1\right) / 2$ shows that $\overline{C_{n}}$ is not $\chi(K)$-good.

When $n$ is odd this is easy. Since we are assuming that $n \geq 8$ then $\left|V\left(K\left(\overline{C_{n}}\right)\right)\right|>2 n+1$ and then there are at least two cliques $Q_{1}$ and $Q_{2}$ of $\overline{C_{n}}$ such that $O\left(Q_{1}\right) \cap O\left(Q_{2}\right)=\emptyset$ and let $S=O\left(Q_{1}\right) \cup O\left(Q_{2}\right)$. Since $n$ is odd then $\left|O\left(Q_{1}\right)\right|$ and $\left|O\left(Q_{2}\right)\right|$ are both odd and each complete of $K\left(\overline{C_{n}}\right)$ contains at most $\left\lfloor\left|O\left(Q_{1}\right)\right| / 2\right\rfloor+\left\lfloor\left|O\left(Q_{2}\right)\right| / 2\right\rfloor=\left(\left|O\left(Q_{1}\right)\right|+\left|O\left(Q_{1}\right)\right|\right) / 2-1=|S| / 2-1$ elements from $S$, and the claim is proved.

Consider the case when $n=4 k+4$ and $k \geq 1$. Let $a_{1}, \ldots, a_{2 k+1}$ be the finite sequence formed by $k$ terms equal to 2 , followed by one 3 , followed by $k-1$ terms equal to 2 , followed by one 3 , and let $Q \subseteq\{0,1, \ldots, n-1\}$ be the set of the partial sums of $\left\{a_{i}\right\}$ modulo $n$, i.e., $Q=\left\{b_{1}, \ldots, b_{2 k+1}\right\}$ where $b_{i}$ is equal to $a_{1}+\cdots+a_{i}$ modulo $n$, for each $i=1,2, \ldots, 2 k+1$ (in particular, $b_{2 k+1}=0$ ). Then $Q$ is a clique of $\overline{C_{n}}$ of period $n$. Let $S=O(Q)$ and let $\mathcal{Q}$ be any complete of $K\left(\overline{C_{n}}\right)$. If $\mathcal{Q} \cap S=\emptyset$ then the claim is trivially true. Without loss of generality $Q \in \mathcal{Q}$. By construction, $Q \cap(Q+2 k+2)=\emptyset$, thus $Q+2 k+2 \notin \mathcal{Q}$. Since $\mathcal{Q}$ does not contain both $Q+j$ and $Q+j+1$ for any $j$ then $\mathcal{Q}$ contains at most $2 k+1=|S| / 2-1$ elements of $S$, as claimed.

Finally, consider the case $n=4 k+6$ for any $k \geq 1$. Let $a_{1}, \ldots, a_{2 k+2}$ be the finite sequence formed by $k$ terms equal to 2 , followed by one 3 , followed by $k$ terms equal to 2 , followed by one 3 , let $b_{1}, \ldots, b_{2 k+2}$ be the sequence formed by $k$ terms equal to 2 , followed by one 3 , followed by $k-1$ terms equal to 2 , followed by one 3 , followed by one 2 , and let $Q_{1}, Q_{2} \subseteq\{0,1, \ldots, n-1\}$ be the partial sums modulo $n$ of $\left\{a_{i}\right\}$ and $\left\{b_{j}\right\}$, respectively. Then $Q_{1}$ and $Q_{2}$ are cliques of $\overline{C_{n}}, Q_{1}$ has period $n / 2=2 k+3$ and $Q_{2}$ has period $n=4 k+6$. Let $S=O\left(Q_{1}\right) \cup O\left(Q_{2}\right)$ and let $\mathcal{Q}$ be any complete of $K\left(\overline{C_{n}}\right)$. It is enough to prove that $|\mathcal{Q} \cap S| \leq 3 k+3$. If $\mathcal{Q} \cap O\left(Q_{2}\right)=\emptyset$ then the claim is trivially true. Without loss of generality $Q_{2} \in \mathcal{Q}$ and then $Q_{2}+1, Q_{2}+2 k+4 \notin \mathcal{Q}$, which implies that 
$\left|\mathcal{Q} \cap O\left(Q_{2}\right)\right| \leq 2 k+2$. Since necessarily $\left|\mathcal{Q} \cap O\left(Q_{1}\right)\right| \leq k+1$, we conclude that $|\mathcal{Q} \cap S| \leq 3 k+3$ and the claim is proved also in this case.

We have the following inclusions:

coordinated $\subset \chi(K)$-perfect $\subset\left(\left\{C_{2 k+1}: k \geq 2\right\} \cup\left\{\overline{C_{n}}: n \geq 7\right\}\right)$-free $\subset$ perfect.

To see that the three inclusions are proper, notice that the 3 -sun is $\chi(K)$ perfect (as it is hereditary $K$-perfect) but not coordinated since $\Delta_{\mathrm{c}}(3$-sun $)=3$ and $\gamma_{\mathrm{c}}(3$-sun $)=4$, and that $S_{2}$ (cf. Figure 10, p. 28) has no odd holes and no antiholes but $K\left(S_{2}\right)$ is not $\chi$-good since $\omega\left(K\left(S_{2}\right)\right)=3$ and $\chi\left(K\left(S_{2}\right)\right)=4$, and finally $\overline{C_{8}}$ proves that the rightmost inclusion is also proper.

As we did with the classes $\mathcal{O}_{1}$ and $\mathcal{O}_{2}$, we propose to classify the minimal forbidden induced subgraphs for the class of coordinated graphs into two classes of obstructions. The class $\mathcal{O}_{3}$ of those graphs $G$ with $\Delta_{\mathrm{c}}(G)<\omega(K(G))$ and the class $\mathcal{O}_{4}$ of those graphs $G$ for which $\omega(K(G))<\chi(K(G))$ holds. Again, Theorem 19 tells us that hereditary clique-Helly obstructions satisfy $\Delta_{c}(G)=$ $\omega(K(G))$, and necessarily belong to the class $\mathcal{O}_{4}$. The obstructions appearing in the partial minimal forbidden induced subgraphs characterizations in the literature belong all to the class $\mathcal{O}_{4}$ with the only exceptions of the 3 -sun, $\overline{2 P_{4}}$ and $\bar{R}$ (cf. Theorem 34).

Finally, note that the $\alpha(K)$-perfect and $\chi(K)$-perfect graphs define classes that are incomparable because $\overline{C_{9}}$ is $\alpha(K)$-perfect but not $\chi(K)$-perfect, and the viking with 7 vertices (cf. Figure 10, p. 28) is $\chi(K)$-perfect but not $\alpha(K)$-perfect. The graph of Figure 6 is $\alpha(K)$-perfect and $\chi(K)$-perfect but not $K$-perfect, and proves the following proper inclusion:

$$
\text { hereditary } K \text {-perfect } \subset \alpha(K) \text {-perfect } \cap \chi(K) \text {-perfect }
$$

\section{$5 \quad$ Partial characterizations}

In this section we review characterizations of clique-perfect and coordinated graphs when restricted to different graph classes. We review the previous results, present some new contributions and formulate the main open problems. Computational complexity issues are also discussed.

The class of clique-perfect graphs is hereditary and thus admits some forbidden induced subgraph characterization. Nevertheless, although some families of forbidden induced subgraphs were identified and some partial characterizations were formulated, a complete list of forbidden induced subgraphs for the class of clique-perfect graphs is not known. Furthermore, the problem of determining the complexity of the recognition of clique-perfect graphs is also open. These two questions are regarded as the main open problems related to clique-perfect graphs (see for instance [10]).

The coordinated graph recognition problem is NP-hard and it is NP-complete even restricted to \{gem, $C_{4}$,odd hole\}-free graphs with $\Delta=4, \omega=3$ and $\Delta_{\mathrm{c}}=3[67]$. The main open problem regarding coordinated graphs is to find the 
complete list of minimal forbidden induced subgraphs. This problem seems to be difficult because in [69] several families of minimally non-coordinated graphs were described whose cardinality grows exponentially on the number of vertices and edges.

Although there are some partial results, the problem of given a characterization by forbidden induced subgraphs of neighborhood-perfect graphs is also open in general. Similarly, although some polynomial-time algorithms for recognizing neighborhood-perfectness when the input graph is known to belong to certain graph classes, the computational complexity of recognizing neighborhoodperfect graphs in general is not known. These are the main open problems regarding neighborhood-perfect graphs.

In this section we present the known partial results on clique-perfect, coordinated graphs, and/or neighborhood-perfect graphs regarding the problems of characterizing by minimal forbidden induced subgraphs and determining the computational complexity of their recognition when restricted to different graph classes. These graph classes are: chordal graphs, diamond-free graphs, $P_{4}$-tidy graphs, Helly circular-arc graphs, complements of forests, line graphs and complements of line graphs, some other subclasses of claw-free graphs and two superclasses of triangle-free graphs.

\subsection{Chordal graphs}

Lehel and Tuza [53] proved that in chordal graphs, balanced and neighborhoodperfect graphs coincide and they are those graphs with no induced odd suns. This, together with Theorem 17, implies the following result.

Theorem $23([53,9])$ Let $G$ be a chordal graph. Then $G$ is clique-perfect if and only if $G$ contains no induced odd sun, if and only if $G$ is balanced, if and only if $G$ is neighborhood-perfect.

Notice that odd suns may properly contain odd suns as induced subgraphs, thus unfortunately this characterization is not by minimal forbidden induced subgraphs. Indeed, it is an open problem to determine the minimal odd suns in the sense of induced subgraphs. As balanced graphs can be recognized in polynomial time, the same algorithm solves, in polynomial time, the problem of recognizing clique-perfect graphs (and thus neighborhood-perfect graphs) restricted to chordal graphs.

Since clique-perfect chordal graphs coincide with balanced chordal graphs then both are subclasses of coordinated chordal graphs. The inclusion is proper since, for instance, the graph displayed in Figure 7 is an example of an odd sun that is coordinated. The fact that clique-perfect chordal graphs are balanced implies, by Theorem 14, that for clique-perfect chordal graphs both parameters $\alpha_{\mathrm{c}}$ and $\tau_{\mathrm{c}}$ (that coincide) can be computed in polynomial time.

Notice that also the 3 -sun is chordal and hereditary $K$-perfect but not cliqueperfect, or coordinated. So hereditary $K$-perfection does not coincide with coordination or clique-perfection or balancedness even when restricted to chordal graphs. 


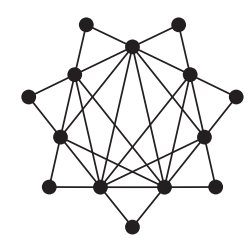

Figure 7: A chordal coordinated odd sun.

\subsection{Diamond-free graphs}

The characterization by forbidden induced subgraphs of those diamond-free graphs that are clique-perfect is the following:

Theorem 24 ([11]) Let $G$ be a diamond-free graph. Then $G$ is clique-perfect if and only if $G$ contains no induced odd generalized sun.

In fact, the authors prove that diamond-free graphs with no odd generalized suns are hereditary clique-Helly and hereditary $K$-perfect, and therefore cliqueperfect.

The problem of deciding whether there exists a polynomial-time algorithm for the recognition of the clique-perfection of a diamond-free graph was left open in [11]. This problem was solved in [15], where it was proved that such an algorithm exists, based on the following result.

Theorem 25 ([15]) Let $G$ be a diamond-free graph. Then $G$ is clique-perfect if and only if $G$ is balanced.

Proof. Since balanced graphs are clique-perfect then we only need to prove that diamond-free clique-perfect graphs are balanced, or equivalently, that a diamond-free graph that is not balanced is not clique-perfect. Assume that $G$ is a diamond-free graph and that $G$ is not balanced. By Theorem $6, G$ contains an unbalanced cycle $C$, that is, an odd cycle $C$ and for each edge $e$ of $C$ a (possibly empty) complete $W_{e} \subseteq N_{G}(e) \backslash C$ such that $N_{C}\left(W_{e}\right) \cap N_{C}(e)=\emptyset$. We claim that $C$ is proper. Suppose, for contradiction, that some edge $e=x y$ of $C$ is improper, and let $v$ in $C$ such that $\{x, y, v\}$ is a triangle of $G$. Since $N_{C}\left(W_{e}\right) \cap N_{C}(e)=\emptyset$, then there is a vertex $w \in W_{e}$ such that $v$ is not adjacent to $w$, and thus $\{v, w, x, y\}$ induces a diamond in $G$, a contradiction. We conclude that $C$ is proper and thus $V(C)$ induces an odd generalized sun. By Theorem $17, G$ is clique-imperfect.

Thus, the problems of recognizing balancedness and recognizing clique-perfection coincide when restricted to diamond-free graphs. Therefore the recognition of clique-perfection can also be solved in polynomial time for diamond-free graphs. Moreover, by Theorems 14 and 25, $\alpha_{\mathrm{c}}$ (and therefore also $\tau_{\mathrm{c}}$ ) can be computed in polynomial time for clique-perfect diamond-free graphs. 


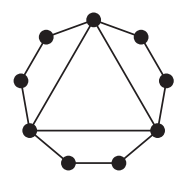

Figure 8: A coordinated diamond-free odd generalized sun.

Notice that if $G$ is a diamond-free graph, the problem of deciding whether $G$ is a minimal odd generalized sun can be solved in polynomial time (it suffices to check that $G$ is not clique-perfect but $G-\{v\}$ is clique-perfect for every vertex $v$ of $G$ ). Surprisingly, the problem of deciding whether $G$ is an odd generalized sun (not necessarily minimal) is NP-complete even if $G$ is a trianglefree graph [50]. Notice that an odd cycle in a triangle-free graph cannot have improper edges. Hence, if $G$ is a triangle-free graph with an odd number of vertices, then $G$ is an odd generalized sun if and only if $G$ has a Hamiltonian cycle, and the Hamiltonian cycle problem on triangle-free graphs with an odd number of vertices is NP-complete [43, pp. 56-60].

Since

balanced graphs $\subset$ hereditary clique-Helly $\cap$ hereditary $K$-perfect $\subset$ clique-perfect

holds and diamond-free graphs are hereditary clique-Helly we conclude, as a corollary of Theorem 25 and its proof, the following.

Corollary 26 Let $G$ be a diamond-free graph. Then the following conditions are equivalent (and can be decided in polynomial time):

1. $G$ is balanced.

2. $G$ is hereditary $K$-perfect.

3. $G$ is clique-perfect.

4. $G$ contains no induced proper odd cycle.

Since diamond-free clique-perfect graphs are balanced then they are also coordinated. Therefore the class of clique-perfect diamond-free graphs is a subclass of the class of coordinated diamond-free graphs. The inclusion is proper since the graph of Figure 8 is coordinated but not clique-perfect.

Odd holes and complete odd suns are minimally clique-imperfect. However, there are other odd generalized suns that contain proper induced odd generalized suns and consequently are not minimally clique-imperfect. The same is true even for proper odd cycles. Thus, the characterizations of Theorem 25 and Corollary 26 are not by minimal forbidden induced subgraphs. The minimal forbidden induced subgraph characterization of clique-perfect (or equivalently balanced) graphs restricted to diamond-free graphs was described in [1]. The corresponding minimal forbidden diamond-free induced subgraphs are the odd holes and the sunoids (which can be put in correspondence with Dyck-paths); the reader is referred to [1] for the details. 


\subsection{Superclasses of cographs}

It is known that comparability graphs are clique-perfect [2]. Since cographs are comparability graphs [42] then cographs are also clique-perfect. In [51] the authors gave a simpler proof of the clique-perfection of cographs based on the following result.

Theorem 27 ([51]) Let $G$ be the join of the graphs $G_{1}$ and $G_{2}$. Then $\alpha_{\mathrm{c}}(G)=$ $\min \left\{\alpha_{\mathrm{c}}\left(G_{1}\right), \alpha_{\mathrm{c}}\left(G_{2}\right)\right\}$ and $\tau_{\mathrm{c}}(G)=\min \left\{\tau_{\mathrm{c}}\left(G_{1}\right), \tau_{\mathrm{c}}\left(G_{2}\right)\right\}$. In particular, $G$ is clique-perfect if and only if $G_{1}$ and $G_{2}$ are clique-perfect.

As a corollary it is possible to compute $\alpha_{\mathrm{c}}(G)$ and $\tau_{\mathrm{c}}(G)$ of a given cograph $G$ in linear time by first computing its cotree [29].

Distance-hereditary graphs and $P_{4}$-tidy graphs define two superclasses of cographs not included in the class of comparability graphs. In [52], distancehereditary graphs were shown to be clique-perfect, relying on a decomposition tree of distance-hereditary graphs introduced in [21]. In [52], linear-time algorithms for computing $\alpha_{\mathrm{c}}(G)$ and $\tau_{\mathrm{c}}(G)$ for any distance-hereditary graph are also presented. In [15], those $P_{4}$-tidy graphs that are clique-perfect were characterized by minimal forbidden induced subgraphs. Moreover, it was shown that the problems of recognizing clique-perfect graphs can be solved in linear time for $P_{4}$-tidy graphs.

Theorem 28 ([15]) Let $G$ be a $P_{4}$-tidy graph. Then $G$ is clique-perfect if and only if $G$ contains neither $C_{5}$ nor 3 -sun as an induced subgraph. Moreover, clique-perfectness of $P_{4}$-tidy graphs can be decided in linear time.

Furthermore, it was shown that both parameters that define clique-perfectness can be computed in linear time for $P_{4}$-tidy graphs.

Theorem 29 ([15]) There are linear-time algorithms that compute $\alpha_{\mathrm{c}}(G)$ and $\tau_{\mathrm{c}}(G)$ for any given $P_{4}$-tidy graph $G$.

The proof of the above theorems relies on the structural characterization of $P_{4}$-tidy graphs given in Theorem 5. Analogous results to the above theorems for neighborhood-perfectness of $P_{4}$-tidy graphs were obtained in [37].

Theorem 30 ([37]) A $P_{4}$-tidy graph $G$ is neighborhood-perfect if and only if $G$ has no induced $\overline{3 K_{2}}, 3$-sun, or $C_{5}$. Moreover, neighborhood-perfectness of $P_{4}$-tidy graphs can be decided in linear time.

Theorem 31 ([37]) There are linear-time algorithms that compute $\alpha_{\mathrm{n}}(G)$ and $\rho_{\mathrm{n}}(G)$ for any given $P_{4}$-tidy graph $G$.

Since the 3 -sun and $C_{5}$ are not coordinated, the class of coordinated $P_{4}$ tidy graphs is included in the class of clique-perfect $P_{4}$-tidy graphs. Moreover, the inclusion is proper since the graph tent $\cup K_{2}$ is $P_{4}$-tidy and clique-perfect (having $\alpha_{\mathrm{c}}=\tau_{\mathrm{c}}=2$ ) but not coordinated (since $\Delta_{\mathrm{c}}=3$ and $\gamma_{\mathrm{c}}=4$ ). The graph 


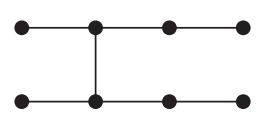

Figure 9: The graph $R$

$\overline{3 K_{2}}$ is an example of a coordinated $P_{4}$-tidy graph that is not neighborhoodperfect; thus, coordinated $P_{4}$-tidy graphs are not contained in neighborhoodperfect $P_{4}$-tidy graphs.

It was proved in [9] that if $F$ is a forest then $\bar{F}$ is clique-perfect. As the class of complements of forests is closed under taking induced subgraphs, it suffices to prove that $\alpha_{\mathrm{c}}(\bar{F})=\tau_{\mathrm{c}}(\bar{F})$. We may assume that $F$ has no isolated vertex, because if $u$ were an isolated vertex of $F$ then every clique of $\bar{F}$ would contain $u$ implying $\alpha_{\mathrm{c}}(\bar{F})=\tau_{\mathrm{c}}(\bar{F})=1$. Thus $F$ has no universal vertex, which means that $\tau_{\mathrm{c}}(\bar{F})>1$. Moreover $F$ has some leaf $u$ and let $v$ be the only neighbor of $u$ in $F$. Then $\{u, w\}$ is a clique-transversal of $\bar{F}$, which shows that $\tau_{\mathrm{c}}(\bar{F})=2$. Furthermore, since every connected component of $F$ is a tree with at least two vertices, $\alpha_{\mathrm{c}}(\bar{F})=\tau_{\mathrm{c}}(\bar{F})=2$. This, together with Theorem 27 immediately implies the following.

Theorem $32([\mathbf{9}, \mathbf{5 1}])$ Every tree-cograph is clique-perfect.

In [37], the following characterization of neighborhood-perfect graphs by forbidden induced subgraphs was proved.

Theorem 33 ([37]) If $G$ is a tree-cograph, then $G$ is neighborhood-perfect if and only if $G$ contains no induced $\overline{3 K_{2}}$ or $P_{6}+3 K_{1}$.

Moreover, from this a linear-time recognition algorithm for neighborhoodperfectness of tree-cographs was also found in [37]. Furthermore, in the same work, linear-time algorithms for computing $\alpha_{\mathrm{n}}(G)$ and $\rho_{\mathrm{n}}(G)$ for any treecograph $G$ were devised. In the following subsection, we will present the characterization of coordinated graphs for complements of forests, a subclass of tree-cographs.

\subsection{Complements of forests}

Recall from the previous subsection that all complements of forests are cliqueperfect. Moreover, it follows from Theorem 33 that those complements of forests that are neighborhood-perfect are those that are 3-pyramid-free. In [18] a characterization by minimal forbidden induced subgraphs of those coordinated graphs within the class of complements of forests was found. Recall that $2 P_{4}$ is the disjoint union of two $P_{4}$ 's and let $R$ be the graph depicted in Figure 9. Both $\overline{2 P_{4}}$ and $\bar{R}$ are not coordinated, since $\Delta_{\mathrm{c}}\left(\overline{2 P_{4}}\right)=\Delta_{\mathrm{c}}(\bar{R})=6$ and $\gamma_{\mathrm{c}}\left(\overline{2 P_{4}}\right)=\gamma_{\mathrm{c}}(\bar{R})=7$. The characterization is as follows.

Theorem 34 ([18]) Let $G$ be the complement of a forest. Then $G$ is coordinated if and only if $G$ contains no induced $\overline{2 P_{4}}$ and no induced $\bar{R}$. 
A characterization of those forests obtained by identifying the false twins of $\bar{G}$ when $G$ is coordinated, called $c$-forest, leads to a linear time recognition algorithm for coordinated graphs within the class of complements of forests [18].

\subsection{Line graphs and complements of line graphs}

The characterization by forbidden induced subgraphs of those line graphs that are clique-perfect is as follows.

Theorem 35 ([10]) Let $G$ be a line graph. Then $G$ is clique-perfect if and only if $G$ has no odd holes and contains no induced 3-sun.

The first part of the proof consists of proving that perfect line graphs are $K$-perfect, and relies on the characterization of perfect line graphs in [58] and on a property of clique-cutsets in perfect graphs [5]. Therefore, those perfect line graphs that are in addition hereditary clique-Helly are also clique-perfect. In the second part of the proof those line graphs that are not hereditary clique-Helly are treated separately. In fact, hereditary $K$-perfection and clique-perfection do not coincide for line graphs as one realizes by considering the 3 -sun.

Those line graphs that are coordinated were characterized by forbidden induced subgraphs in [18].

Theorem 36 ([18]) Let $G$ be a line graph. Then $G$ is coordinated if and only if $G$ has no odd hole and contains no induced 3-sun.

Reasoning as in the first part of the proof of Theorem 35, line graphs without odd holes are $K$-perfect, and then those that in addition are hereditary cliqueHelly, are coordinated. Therefore the proof consists of studying those line graphs that have no odd holes but have some family of cliques that does not satisfy the Helly property.

Notice that by Theorems 35 and 36, coordinated and clique-perfect graphs coincide when restricted to line graphs.

In [18] also a characterization of coordinated line graphs in terms of its root graph is given. To formulate it, the authors introduce the following definitions. Given a graph $H$ and a set $S \subseteq V(H)$, denote by $E_{H}(S)$ the set of edges of $H$ that have both endpoints in $S$. The set $S \subseteq V(H)$ is an edge separator of $H$ if every vertex of $S$ belongs to a different connected component of $G \backslash E_{H}(S)$. Let $t=\left\{v_{1}, v_{2}, v_{3}\right\}$ be a triangle of a graph $H$. The triple $\left(v_{1}, v_{2}, v_{3}\right)$ is a well ordering of $t$ if $d_{H}\left(v_{1}\right) \leq d_{H}\left(v_{2}\right) \leq d_{H}\left(v_{3}\right)$ and at least one of the following conditions holds: $(i) d_{H}\left(v_{1}\right)<d_{H}\left(v_{2}\right)$, or $(i i) N_{H}\left[v_{3}\right]$ is equal to both $N_{H}\left[v_{1}\right]$ and $N_{H}\left[v_{2}\right]$, or (iii) $N_{H}\left[v_{3}\right]$ is equal to none of $N_{H}\left[v_{1}\right]$ and $N_{H}\left[v_{2}\right]$. Every triangle admits some well ordering permutation [18]. Let $E_{t}$ be the set of edges $\left\{v_{1} v_{2}, v_{2} v_{3}, v_{3} v_{1}\right\}$, and if $\mathcal{T}$ is a family of triangles, let $E_{\mathcal{T}}=\bigcup_{t \in \mathcal{T}} E_{t}$. With this terminology, the characterization can now be formulated as follows:

Theorem 37 ([18]) Let $H$ be a graph and $\mathcal{T}$ be the set of triangles of $H$. Then the following statements are equivalent: 
1. $L(H)$ is coordinated.

2. $H \backslash E_{\mathcal{T}}$ is bipartite and every well ordered triangle $\left(v_{1}, v_{2}, v_{3}\right)$ of $H$ satisfies one of the following statements:

(a) $d_{H}\left(v_{1}\right)=2$ and $N_{H}\left[v_{2}\right] \cap N_{H}\left[v_{3}\right]$ is an edge separator of $H$.

(b) $d_{H}\left(v_{1}\right)=3, v_{1}$ and $v_{2}$ are true twins and $N_{H}\left[v_{1}\right]$ is an edge separator of $G$.

This characterization leads to a linear-time recognition algorithm for coordinated graphs within the class of line graphs. As a corollary, a linear-time recognition algorithm for clique-perfect graphs within this class follows. In [18], a linear-time algorithm for determining $\Delta_{\mathrm{c}}(G)$ and $\gamma_{\mathrm{c}}(G)$ for any coordinated line graph $G=L(H)$ is also presented.

We would like to remark that the first part of the proof of Theorem 36 implies the following: a line graph is hereditary $K$-perfect if and only if it has no odd holes, if and only if it is perfect.

In [16], clique-perfectness of complements of line graphs was characterized by minimal forbidden induced subgraphs.

Theorem 38 ([16]) If $G$ is the complement of a line graph, then $G$ is cliqueperfect if and only if $G$ contains no induced 3 -sun and has no antihole $\overline{C_{k}}$ for any $k \geq 5$ such that $k$ is not a multiple of 3 .

Let $G$ be the complement of the line graph of a graph $H$. In order to prove the above theorem, the parameters $\alpha_{\mathrm{c}}(G)$ and $\tau_{\mathrm{c}}(G)$ are expressed in terms of the graph $H$. Clearly, the cliques of $G$ are precisely the maximal matchings of $H$. The matching-transversal number of $H$, denoted by $\tau_{\mathrm{m}}(H)$, is defined as the minimum size of a set of edges of $H$ that meets every maximal matching of $H$. Similarly, the matching-independence number of $H$, denoted by $\alpha_{\mathrm{m}}(H)$, is defined as the maximum size of a set of pairwise-disjoint maximal matchings of $H$. A graph is called matching-perfect [16] if $\tau_{\mathrm{m}}(H)=\alpha_{\mathrm{m}}(H)$ for every subgraph (induced or not) of $H$. It is not hard to see that $G$ is clique-perfect if and only if $H$ is matching-perfect. In fact, the proof of Theorem 38 follows as a corollary of the following characterization of matching-perfect graphs.

Theorem 39 ([16]) A graph $H$ is matching-perfect if and only if $H$ contains no bipartite claw and the length of each cycle of $H$ is at most 4 or a multiple of 3 .

In its turn, Theorem 39 is proved by means of a decomposition theorem describing in detail the linear and circular structure of graphs containing no bipartite claw proved in [16].

Notice that since the 3 -sun and antiholes different from $\overline{C_{6}}$ are neither coordinated nor neighborhood-perfect, coordinated (resp. neighborhood-perfect) complements of line graphs are clique-perfect. Moreover, these inclusion are proper and coordination and neighborhood-perfectness differ for complements of line graphs. In fact, the 3-pyramid $=\overline{L\left(K_{4}\right)}$ is clique-perfect and coordinated but not neighborhood-perfect, whereas $\overline{2 P_{4}}=\overline{L\left(2 P_{5}\right)}$ is clique-perfect and neighborhood-perfect but not coordinated. 


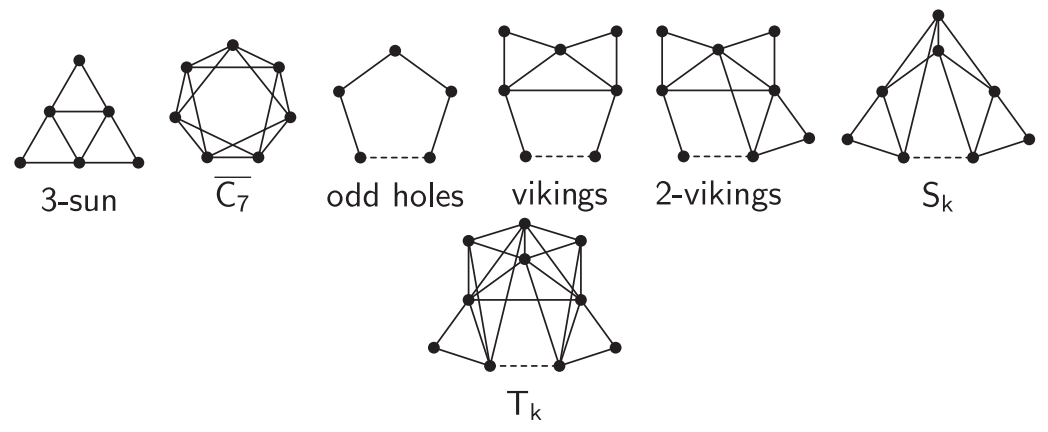

Figure 10: Minimal forbidden induced subgraphs for clique-perfect graphs inside the class of Helly circular-arc graphs. Dashed lines represent induced paths of length $2 k-3$ for each $k \geq 2$.

\subsection{Helly circular-arc graphs}

In [11] a characterization of those Helly circular-arc graphs that are cliqueperfect was formulated in terms of forbidden induced subgraphs. The forbidden induced subgraphs are displayed in Figure 10. The 3-sun, odd holes, vikings and 2-vikings are all odd generalized suns. The authors show that, within Helly circular-arc graphs, there are only two families of minimally clique-imperfect graphs which are not odd generalized suns or antiholes, namely, $S_{k}$ and $T_{k}$ for each $k \geq 2$. Since the graphs of Figure 10 do not contain properly each other as induced subgraphs then the following is a characterization by minimal forbidden induced subgraphs:

Theorem 40 ([11]) Let $G$ be a Helly circular-arc graph. Then $G$ is cliqueperfect if and only if it does not contain a 3-sun, an antihole of length 7 , an odd hole, a viking, a 2-viking or one of the graphs $S_{k}$ or $T_{k}$ for each $k \geq 2$.

Whether a graph is a Helly circular-arc graph can be decided in linear time and, if affirmative, both parameters $\alpha_{\mathrm{c}}(G)$ and $\tau_{\mathrm{c}}(G)$ can also be computed in linear time $[34,35,55]$. However, these facts do not immediately imply the existence of a polynomial-time recognition algorithm for clique-perfect Helly circular-arc graphs (because we need to verify the equality for every induced subgraph). The characterization given in [11] leads to such an algorithm, which is strongly based on the recognition of perfect graphs [23]. The idea of the algorithm is similar to the one used in [28] for recognizing balanceable matrices. It was proved in [75] that clique-perfectness coincides with neighborhoodperfectness for Helly circular-arc graphs. As in [11], the proof strategy consists in studying separately those Helly circular-arc graphs that are hereditary cliqueHelly from those that are not so.

Theorem 41 ([75]) If $G$ is a Helly circular-arc graph, then $G$ is clique-perfect if and only if $G$ is neighborhood-perfect. 
Coordinated Helly circular-arc graphs and clique-perfect Helly circular-arc graphs are not comparable. In fact, on the one hand, for each $k \geq 2$, the viking with $2 k+3$ vertices is coordinated but not clique-perfect (since $\alpha_{\mathrm{c}}=k$ and $\tau_{\mathrm{c}}=k+1$ ), and on the other hand, the graph $\bar{R}$ (cf. Figure 9 ) is an example of a Helly circular-arc graph which is clique-perfect but not coordinated (since $\Delta_{\mathrm{c}}=6$ and $\left.\gamma_{\mathrm{c}}=7\right)$.

\subsection{Some subclasses of claw-free graphs}

Another class where clique-perfectness, coordination and neighborhood-perfectness coincide is the class of hereditary clique-Helly claw-free graphs.

Theorem 42 ([10]) Let $G$ be a hereditary clique-Helly claw-free graph. Then $G$ is clique-perfect if and only if no induced subgraph of $G$ is an odd hole or an antihole of length $\%$.

It is well-known that for hereditary clique-Helly graphs, each clique has some proper edge; i.e., an edge that belongs to that clique only. Moreover, it was proved in [53] that every graph $G$ for which each clique has a proper edge satisfies $\alpha_{\mathrm{c}}(G)=\alpha_{\mathrm{n}}(G)$ and $\tau_{\mathrm{c}}(G)=\rho_{\mathrm{n}}(G)$. Thus, the following holds.

Theorem 43 ([53]) A hereditary clique-Helly graph $G$ is clique-perfect if and only if $G$ is neighborhood-perfect.

The proof of Theorem 42 relies on the decomposition theorem for claw-free graphs that appears in [25]. Indeed, in [10] it is proved more, that hereditary clique-Helly claw-free graphs with no odd holes and no induced $\overline{C_{7}}$ are $K$-perfect. Since, conversely, odd holes and $\overline{C_{7}}$ are not $K$-perfect, then, by Theorem 42 , Corollary 9, Theorem 21 and Theorem 43, the following set of equivalencies follow.

Corollary 44 ([10]) Let $G$ be a hereditary clique-Helly claw-free graph. Then the following assertions are equivalent:

1. $G$ is clique-perfect.

2. $G$ is hereditary K-perfect.

3. $G$ is coordinated.

4. $G$ is neighborhood-perfect.

5. $G$ is perfect.

6. G has no odd holes and contains no induced $\overline{C_{7}}$.

Recall that deciding whether a graph is perfect is solvable in polynomial time [23].

Relying on Theorem 42, the following characterization of those claw-free planar graph that are clique-perfect was found in [54].

Theorem 45 ([54]) Let $G$ be a claw-free graph such that $G$ is planar or $\Delta(G) \leq$ 4. Then, $G$ is clique-perfect if and only if $G$ contains no odd hole and neither a 3-sun nor the graph of Figure 11 as an induced subgraph. 


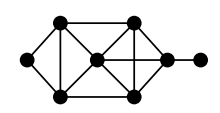

Figure 11: A minimal forbidden induced subgraph for the class of clique-perfect graphs

As an immediate consequence, they observe that every cubic claw-free graph is clique-perfect.

\subsection{Superclasses of triangle-free graphs}

Triangle-free graphs were extensively studied in the literature, usually in the context of graph coloring problems (see for instance $[48,59,62]$ ). It is easy to see that if a graph $G$ is triangle-free, then $G$ is perfect if and only if $G$ is cliqueperfect, if and only if $G$ is coordinated, because bipartite graphs are perfect, clique-perfect, and coordinated.

In [17], these equivalencies were extended to two superclasses of triangle-free graphs: paw-free and $\left\{\right.$ gem, $W_{4}$, bull $\}$-free graphs.

Theorem 46 ([17]) Let $G$ be a paw-free graph. The following statements are equivalent:

1. $G$ is perfect.

2. $G$ is clique-perfect.

3. $G$ is coordinated.

4. $G$ does not contain odd holes.

For paw-free graphs, not every perfect graph is hereditary $K$-perfect. In [68], it is proved that if $G$ is a paw-free graph having at least three anticomponents and each anticomponent of $G$ has at least 3 vertices, then $K(G)$ contains an induced $C_{5}$. In particular, $K\left(\overline{3 K_{3}}\right)$ contains an induced $C_{5}$, so it is not perfect. Moreover, the following theorem is proved.

Theorem 47 [68] Let $G$ be a perfect connected paw-free graph. Then the following statements are equivalent:

1. $G$ is $K$-perfect.

2. $K(G)$ does not contain an induced $C_{5}$.

3. Either $G$ is bipartite or at least one anticomponent of $G$ has at most two vertices.

As a corollary of Theorem 47 (and reasoning as in the proof of Theorem 46) the following characterizations of hereditary $K$-perfect paw-free graphs can be obtained:

Corollary 48 Let $G$ be a paw-free graph. Then the following conditions are equivalent: 
1. $G$ is hereditary K-perfect.

2. $G$ has no odd holes and contains no induced $\overline{3 K_{3}}$.

3. Each connected component of $G$ is either bipartite or not anticonnected and, in the latter case, at most two of its anticomponents have more than two vertices.

For $\left\{\right.$ gem, $W_{4}$, bull $\}$-free graphs instead, all the discussed main notions coincide. Notice that $\left\{\right.$ gem, $W_{4}$, bull $\}$-free graphs are hereditary clique-Helly by Theorem 4 and recall Theorem 43 .

Theorem $49([\mathbf{1 7}])$ Let $G$ be a $\left\{\right.$ gem, $W_{4}$, bull $\}$-free graph. Then the following statements are equivalent:

1. $G$ is perfect.

2. $G$ is clique-perfect.

3. $G$ is coordinated.

4. $G$ is neighborhood-perfect.

5. $G$ is hereditary K-perfect.

6. $G$ does not contain odd holes.

Using this characterization and the fact that perfect graphs can be recognized in polynomial time, the clique-perfect recognition problem restricted to the class of $\left\{\right.$ gem, $W_{4}$, bull $\}$-free graphs can be solved in polynomial time.

The major step to verify Theorem 49 is to prove that $\left\{\right.$ gem, $W_{4}$, bull $\}$-free graphs without odd holes are $K$-perfect. Since the class of $\left\{\right.$ gem, $W_{4}$, bull,odd hole $\}$-free graphs is hereditary then it follows that they are hereditary $K$-perfect. Since $\left\{\right.$ gem,$W_{4}$, bull $\}$-free graphs are hereditary clique-Helly then the remaining implications follow by Corollary 9 .

In [17] it is mentioned as an interesting open problem to determine the 'biggest' superclass of triangle-free graphs where clique-perfect and coordinated graphs are equivalent.

\section{$6 \quad$ Final remarks}

We would like to put forward some possibilities to study these classes further. Take for instance the case of clique-perfect graphs. We feel that there may be still many opportunities for finding more obstructions to clique-perfection. Indeed, the larger family of obstructions for clique-perfection that are clearly identified in the literature are those that correspond to odd generalized suns, and Table 1 suggests that they represent only a restricted portion of the total set of obstructions.

In most of the cases the approach taken to obtain the partial characterization of clique-perfection (and coordination) reviewed in Sec. 5 depend heavily on the equality between clique-perfection and hereditary $K$-perfection (at least when the graphs are further restricted to be hereditary clique-Helly). So one of the main parts of these characterizations consists on studying how the imperfection 


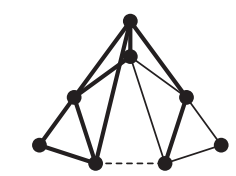

$\mathrm{S}_{\mathrm{k}}$ (with a partial viking in bold)

Figure 12: A viking as a partial subgraph of $S_{k}$.

of $K(G)$ translates into a forbidden structure on $G$. This part of the proof differs radically when restricting to different graph classes. So it would represent a progress to find a way to handle this situation more generally. Notice that by the SPGT, if $K(G)$ is imperfect then $K(G)$ or $\overline{K(G)}$ contains an odd hole. Consider the case when $K(G)$ contains a hole $Q_{1}, \ldots, Q_{n}$ of cliques of $G$. By taking a vertex in each consecutive pair of cliques a cycle $C$ arises in $G$. It happens, as one may suspect, that many possibilities emerge by combining presence of some adjacencies and absence of others. At this point we could think about grouping the arising possibilities together by similarity and a tool that can help to achieve this is the concept of trigraph [22]. A trigraph is a generalization of a graph were some adjacencies are set to be present, some adjacencies are set to be not present, but the remaining adjacencies can be freely set to be present or not. That is, a trigraph represent the whole family of graphs resulting from deciding the presence or not of each of the undecided edges. This could help to reduce the number of forbidden structures into consideration, which Table 1 suggests grows fast. Notice that there are still minimally clique-imperfect graphs that are hereditary $K$-perfect so that their clique graphs do not have odd holes or odd antiholes, this indicates that to describe the full set of obstructions we may need to study also some cycles in the clique graph that do not correspond to odd holes or antiholes.

Further possibilities of attack may arise by classifying the obstructions according to the underlying structure of the odd holes of $K(G)$. This structure may resemble the structure of hypomatchable graphs, which can be constructed from odd holes by attaching paths each of which creates a new hole (see [57] for details). A similitude is observed when we look at the clique graph as extension of the line graph, since for a hypomatchable graph, the graphs obtained by the removal of any vertex admit a perfect matching, that is exactly a stable set in the line graph that, in addition, is a vertex cover. Notice that in a minimally clique-imperfect graph from the set of obstructions $\mathcal{O}_{1}$, the removal of a single vertex produces a graph such that its clique graph admits a stable set and a vertex cover of the same size.

In the same spirit, there is an interesting fact that shows up if we take a closer look at the graphs of Figure 10. For instance, we could consider $S_{k}$ as a merging of two vikings. Figure 12 shows a partial subgraph of $S_{k}$ that is a viking, and other candidates can be found symmetrically. Contrary to what one could expect the clique graph of $S_{k}$ contains just one odd hole and not two odd holes that share some edges. It would be interesting to generalize this or find 
new merging procedure as they may allow us to classify certain obstructions as nonbasic and concentrate on the more restricted basic ones that do not admit such decompositions.

Furthermore, it is interesting to find more general characterizations of hereditary $K$-perfect graphs, as well as characterizing $\alpha(K)$-perfect and $\chi(K)$-perfect graphs.

\section{Acknowledgements}

F. Bonomo, G. Durán and M. Safe were partially supported by ANPCyT PICT 2015-2218 and the UBACyT Grant 20020130100808BA (Argentina). G. Durán was partially supported by FONDECyT Grant 1080286 (Chile) and Millennium Science Institute "Complex Engineering Systems" (Chile). M. Safe was partially supported by CONICET PIO 14420140100027CO and Universidad Nacional del Sur Grant PGI 24/ZL16 (Argentina).

\section{References}

[1] N. Apollonio, A. Galluccio, Minimally unbalanced diamond-free graphs and Dyck-paths, SIAM J. Discrete Math. 29 (4) (2015) 1837-1863.

[2] V. Balachandran, P. Nagavamsi, C. Pandu Rangan, Clique transversal and clique independence on comparability graphs, Inform. Process. Lett. 58 (4) (1996) 181-184.

[3] H.-J. Bandelt, H. M. Mulder, Distance-hereditary graphs, J. Combin. Theory Ser. B 41 (2) (1986) 182-208.

[4] C. Berge, Balanced matrices, Math. Program. 2 (1) (1972) 19-31.

[5] C. Berge, Graphs and Hypergraphs, North-Holland, Amsterdam, 1985.

[6] C. Berge, Hypergraphs: combinatorics of finite sets, vol. 45 of NorthHolland Mathematical Library, North-Holland, Amsterdam, 1989.

[7] C. Berge, M. Las Vergnas, Sur un théorème du type König pour hypergraphes, Ann. N.Y. Acad. Sci. 175 (1970) 32-40.

[8] F. Bonomo, Sobre grafos balanceados y complejidad computacional de problemas asociados a la teoría de grafos perfectos (in Spanish), Master's thesis, Departmento de Matemática, FCEyN, Universidad de Buenos Aires, Buenos Aires, Argentina (2002).

[9] F. Bonomo, On subclasses and variations of perfect graphs, Ph.D. thesis, Departmento de Computación, FCEyN, Universidad de Buenos Aires, Buenos Aires, Argentina (2005). 
[10] F. Bonomo, M. Chudnovsky, G. Durán, Partial characterizations of cliqueperfect graphs I: subclasses of claw-free graphs, Discrete Appl. Math. 156 (7) (2008) 1058-1082.

[11] F. Bonomo, M. Chudnovsky, G. Durán, Partial characterizations of cliqueperfect graphs II: diamond-free and Helly circular-arc graphs, Discrete Math. 309 (11).

[12] F. Bonomo, G. Durán, M. Groshaus, Coordinated graphs and clique graphs of clique-Helly perfect graphs, Util. Math. 72 (2007) 175-191.

[13] F. Bonomo, G. Durán, M. Groshaus, J. L. Szwarcfiter, On clique-perfect and K-perfect graphs, Ars Combin. 80 (2006) 97-112.

[14] F. Bonomo, G. Durán, M. C. Lin, J. L. Szwarcfiter, On balanced graphs, Math. Program. 105 (2-3) (2006) 233-250.

[15] F. Bonomo, G. Durán, M. D. Safe, A. K. Wagler, Clique-perfectness and balancedness of some graph classes, Int. J. Comput. Math. 91 (10) (2014) $2118-2141$.

[16] F. Bonomo, G. Durán, M. D. Safe, A. K. Wagler, Clique-perfectness of complements of line graphs, Discrete Appl. Math. 186 (2015) 19-44.

[17] F. Bonomo, G. Durán, F. Soulignac, G. Sueiro, Partial characterizations of clique-perfect and coordinated graphs: superclasses of triangle-free graphs (2009).

[18] F. Bonomo, G. Durán, F. Soulignac, G. Sueiro, Partial characterizations of coordinated graphs: line graphs and complements of forests (2009).

[19] A. Brandstädt, V. D. Chepoi, F. F. Dragan, Clique $r$-domination and clique $r$-packing problems on dually chordal graphs, SIAM J. Discrete Math. 10 (1) (1997) 109-127.

[20] M. Chang, M. Farber, Z. Tuza, Algorithmic aspects of neighbourhood numbers, SIAM J. Discrete Math. 6 (1) (1993) 24-29.

[21] M.-S. Chang, S.-Y. Hsieh, G.-H. Chen, Dynamic programming on distancehereditary graphs, Lect. Notes Comput. Sci. 1350 (1997) 344-353.

[22] M. Chudnovsky, Berge trigraphs, J. Graph Theory 53 (1) (2006) 1-55.

[23] M. Chudnovsky, G. P. Cornuéjols, X. Liu, P. D. Seymour, K. Vušković, Recognizing Berge graphs, Combinatorica 25 (2) (2005) 143-186.

[24] M. Chudnovsky, N. Robertson, P. D. Seymour, R. Thomas, The Strong Perfect Graph Theorem, Ann. Math. 164 (1) (2006) 51-229.

[25] M. Chudnovsky, P. D. Seymour, The structure of claw-free graphs, London Math. Soc. Lecture Note Ser. 327 (2005) 153-171. 
[26] V. Chvátal, On certain polytopes associated with graphs, J. Combin. Theory Ser. B 18 (2) (1975) 138-154.

[27] M. Conforti, G. P. Cornuéjols, A. Kapoor, K. Vušković, Balanced 0, \pm 1 matrices II. Recognition algorithm, J. Combin. Theory Ser. B 81 (2) (2001) 275-306.

[28] M. Conforti, G. Zambelli, Recognizing balanceable matrices, Math. Program. 105 (2-3) (2006) 161-179.

[29] D. G. Corneil, H. Lerchs, L. Stewart Burlingham, Complement reducible graphs, Discrete Appl. Math. 3 (3) (1981) 163-174.

[30] E. Dahlhaus, P. D. Manuel, M. Miller, Maximum $h$-colourable subgraph problem in balanced graphs, Inform. Process. Lett. 65 (6) (1998) 301-303.

[31] R. P. Dilworth, A decomposition theorem for partially ordered sets, Ann. Math. 51 (1950) 161-166.

[32] F. F. Dragan, Centers of graphs and the Helly property (in Russian), Ph.D. thesis, Universitatea de Stat din Moldova, Chişinău, Moldova (1989).

[33] P. Duchet, Hypergraphs, in: R. L. Graham, M. Grötschel, L. Lovász (eds.), Handbook of Combinatorics, chap. 7, Elsevier, Amsterdam, 1995, pp. 381432.

[34] G. Durán, M. C. Lin, S. Mera, J. L. Szwarcfiter, Algorithms for cliqueindependent sets on subclasses of circular-arc graphs, Discrete Appl. Math. 154 (13) (2006) 1783-1790.

[35] G. Durán, M. C. Lin, S. Mera, J. L. Szwarcfiter, Algorithms for finding clique-transversals of graphs, Ann. Oper. Res. 157 (1) (2008) 37-45.

[36] G. Durán, M. C. Lin, J. L. Szwarcfiter, On clique-transversal and cliqueindependent sets, Ann. Oper. Res. 116 (1) (2002) 71-77.

[37] G. Durán, M. D. Safe, X. S. Warnes, Neighborhood covering and independence on $P_{4}$-tidy graphs and tree-cographs, Ann. Oper. Res. (2017) $32 \mathrm{pp}$. In press.

[38] P. Erdős, T. Gallai, Z. Tuza, Covering the cliques of a graph with vertices, Discrete Math. 108 (1-3) (1992) 279-289.

[39] M. Farber, Characterizations of strongly chordal graphs, Discrete Math. $43(2-3)(1983)$ 173-189.

[40] L. R. Ford, D. R. Fulkerson, Flows in Networks, Princeton University Press, Princeton, NJ, 1962.

[41] D. R. Fulkerson, A. J. Hoffman, R. Oppenheim, On balanced matrices, in: M. Balinski (ed.), Pivoting and Extensions: In honor of A.W. Tucker, vol. 1 of Math. Program. Study, North-Holland, Amsterdam, 1974, pp. 120-133. 
[42] T. Gallai, Transitiv orientierbare Graphen, Acta Math. Acad. Sci. Hung. $18(1-2)(1967) 25-66$.

[43] M. R. Garey, D. S. Johnson, Computers and Intractability: A Guide to the Theory of NP-Completeness, Freeman and Company, San Francisco, 1979.

[44] F. Gavril, Algorithms on circular-arc graphs, Networks 4 (1974) 357-369.

[45] V. Giakoumakis, F. Roussel, H. Thuillier, On $P_{4}$-tidy graphs, Discrete Math. Theor. Comput. Sci. 1 (1997) 17-41.

[46] M. C. Golumbic, Algorithmic Graph Theory and Perfect Graphs, Academic Press, New York, 1980.

[47] V. Guruswami, C. Pandu Rangan, Algorithmic aspects of clique-transversal and clique-independent sets, Discrete Appl. Math. 100 (3) (2000) 183-202.

[48] G. Jin, Triangle-free four-chromatic graphs, Discrete Math. 145 (1-3) (1995) 151-170.

[49] D. Kőnig, Über Graphen und ihre Anwendung auf Determinantentheorie und Mengenlehre, Math. Ann. 77 (1916) 453-465.

[50] A. Korenchendler, personal communication (2007).

[51] A. Lakshmanan S., A. Vijayakumar, On the clique-transversal number of a graph, manuscript (2006).

[52] C.-M. Lee, M.-S. Chang, Distance-hereditary graphs are clique-perfect, Discrete Appl. Math. 154 (3) (2006) 525-536.

[53] J. Lehel, Z. Tuza, Neighborhood perfect graphs, Discrete Math. 61 (1) (1986) 93-101.

[54] Z. Liang, E. Shan, L. Kang, Clique-perfectness of claw-free planar graphs, Graphs Combin. 32 (6) (2016) 2551-2562.

[55] M. C. Lin, J. L. Szwarcfiter, Characterizations and linear time recognition of Helly circular-arc graphs, Lect. Notes Comput. Sci. 4112 (2006) 73-82.

[56] L. Lovász, Normal hypergraphs and the perfect graph conjecture, Discrete Math. 2 (3) (1972) 253-267.

[57] L. Lovász, A note on factor critical graphs, Studia Sci. Math. Hungar. 7 (1972) 279-280.

[58] F. Maffray, Kernels in perfect line-graphs, J. Combin. Theory Ser. B 55 (1992) 1-8.

[59] F. Maffray, M. Preissmann, On the NP-completeness of the $k$-colorability problem for triangle-free graphs, Discrete Math. 162 (1996) 313-317. 
[60] K. Menger, Zur allgemeinen Kurventheorie, Fund. Math. 10 (1927) 96-115.

[61] J. Mycielski, Sur le coloriage des graphs, Colloq. Math. 3 (2) (1955) 161162.

[62] A. Nilli, Triangle-free graphs with large chromatic numbers, Discrete Math. $211(1-3)(2000)$ 261-262.

[63] M. W. Padberg, Characterizations of totally unimodular, balanced and perfect matrices, Tech. Rep. 7423 - OR, Institut für Ökonometrie und Operations Research, Universität Bonn (1974).

[64] E. Prisner, Hereditary clique-Helly graphs, J. Combin. Math. Combin. Comput. 14 (1993) 216-220.

[65] D. J. Rose, R. E. Tarjan, G. S. Lueker, Algorithmic aspects of vertex elimination on graphs, SIAM J. Comput. 5 (1976) 266-283.

[66] M. D. Safe, On structural characterizations of graph classes related to perfect graphs and the König property, Ph.D. thesis, Departamento de Computación, FCEyN, Universidad de Buenos Aires (2011).

[67] F. Soulignac, G. Sueiro, NP-hardness of the recognition of coordinated graphs, in: Ann. XIII CLAIO, Montevideo, Uruguay, 2006.

[68] F. Soulignac, G. Sueiro, Sobre caracterizaciones parciales de grafos coordinados (in Spanish), Master's thesis, Departamento de Computación, FCEyN, Universidad de Buenos Aires (2006).

[69] F. Soulignac, G. Sueiro, Exponential families of minimally non-coordinated graphs, Rev. Un. Mat. Argentina 50 (1) (2009) 75-85.

[70] J. P. Spinrad, Transitive orientation in $O\left(n^{2}\right)$ time, in: STOC '83: Proceedings of the fifteenth annual ACM symposium on Theory of computing, ACM, 1983.

[71] J. L. Szwarcfiter, Recognizing clique-Helly graphs, Ars Combin. 45 (1997) $29-32$.

[72] G. Tinhofer, Strong tree-cographs are birkhoff graphs, Discrete Appl. Math. 22 (3) (1988) 275-288.

[73] S. Tsukiyama, M. Idle, H. Ariyoshi, Y. Shirakawa, A new algorithm for generating all the maximal independent sets, SIAM J. Comput. 6 (3) (1977) $505-517$.

[74] W. D. Wallis, G.-H. Zhang, On maximal clique irreducible graphs, J. Combin. Math. Combin. Comput. 8 (1990) 187-193.

[75] X. Warnes, Structural and algorithmic results on neighborhood-perfect graphs and neighborhood numbers, Master's thesis, Departamento de Matemática, FCEyN, Universidad de Buenos Aires (2014). 
[76] H. Whitney, Congruent graphs and the connectivity of graphs, Amer. J. Math. 54 (1932) 150-168.

[77] G. Zambelli, A polynomial recognition algorithm for balanced matrices, J. Combin. Theory Ser. B 95 (1) (2005) 49-67. 